\title{
Breeding for dual-purpose wheat varieties using marker-trait associations for biomass yield and quality traits
}

Malik, Pernille Louise; Janss, Luc; Nielsen, Linda K.; Borum, Finn; Jørgensen, Henning; Eriksen, Birger; Schjørring, Jan K.; Rasmussen, Søren Kjærsgaard

\section{Published in:}

Theoretical and Applied Genetics

DOI:

10.1007/s00122-019-03431-z

Publication date:

2019

Document version

Peer reviewed version

Citation for published version (APA):

Malik, P. L., Janss, L., Nielsen, L. K., Borum, F., Jørgensen, H., Eriksen, B., Schjørring, J. K., \& Rasmussen, S. K. (2019). Breeding for dual-purpose wheat varieties using marker-trait associations for biomass yield and quality traits. Theoretical and Applied Genetics, 132, 3375-3398. https://doi.org/10.1007/s00122-019-03431-z 
Published version: P. L. Malik, L. Janss, L. K. Nielsen, F. Borum, H. Jørgensen, B. Eriksen, J. K. Schjoerring, S. K. Rasmussen: Breeding for dual-purpose wheat varieties using marker-trait associations for biomass yield and quality traits. Theoretical and Applied Genetics. 132, Issue 12, pp 3375 -3398 (2019) DOI: 10.1007/s00122-019-03431-z

\section{Breeding for dual-purpose wheat varieties using marker-trait associations for biomass yield and quality traits}

Pernille L. Malik , Luc Janss ${ }^{\mathrm{b}}$, Linda K. Nielsenc, Finn Borumc, Henning Jørgensenª Birger Eriksenc, Jan K. Schjoerring ${ }^{\mathrm{a}}$, Søren K. Rasmussen ${ }^{\mathrm{a}^{*}}$

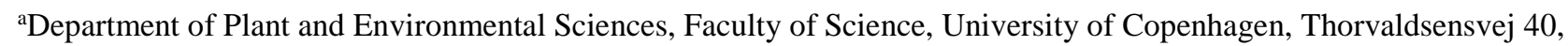
1871 Frederiksberg, Denmark

${ }^{b}$ Department of Molecular Biology and Genetics, Aarhus University, Blichers Alle 20, 8830 Tjele, Denmark 'Sejet Plant Breeding, Nørremarksvej 67, 8700 Horsens, Denmark

${ }^{*}$ Corresponding author: skr@plen.ku.dk, Phone +45 35333436

ORCID iD: 0000-0002-2852-3298 (JKS), 0000-0001-6169-2504 (SKR)

\section{Abstract}

Wheat straw is a valuable feedstock for bioethanol production, but due to the recalcitrant nature of lignocellulose, its efficient use in biorefineries is limited by its low digestibility and difficult conversion of structural carbohydrates into free sugars. A genome-wide association study (GWAS) was conducted to search for significant SNP markers that could be used in a breeding program to improve the value of wheat straw in a biorefinery setting. As part of a three-year breeding program (2015-2017), 190 winter wheat lines were phenotyped for traits that affect the yield and quality of the harvested biomass. These traits included straw yield, plant height, lodging at three growth stages, and Septoria tritici blotch (STB) susceptibility. Release of glucose, xylose and arabinose was determined after hydrothermal pretreatment and enzymatic hydrolysis of the straw. The lines were genotyped using 15K SNP markers and 5552 SNP markers could be used after filtering. Heritability for all traits ranged from 0.02 to 0.74 . GWASs were conducted using CMLM, SUPER and FarmCPU algorithms, to analyse which algorithm could detect the highest number of marker-trait associations (MTAs). Comparable tendencies were obtained from CMLM and FarmCPU, but FarmCPU produced the most significant results. MTAs were obtained for lodging, harvest index, plant height, STB, glucose, xylose and arabinose at a significance level of $\mathrm{p}<9.01 \times 10^{-6}$. MTAs in chromosome $6 \mathrm{~A}$ were observed for glucose, xylose and arabinose, and could be of importance for increasing sugar release for bioethanol production.

Keywords: GWAS, harvest index, lodging, saccharification, Septoria tritici blotch, winter wheat (Triticum aestivum)

Author contributions: P.L.M., J.K.S., B.E. and S.K.R. conceived and designed the experiments; L.K.N., F.B. and B.E. designed and carried out the field experiments, conducted phenotyping and provided SNP marker data; P.L.M. performed all the experiments; P.L.M. analysed the data with the contribution of L.J.; P.L.M. drafted and finalized the article with writing contributions from all the authors; the final article was approved by all the authors.

Key message: This study demonstrates that an active breeding nursery with rotation can be used to identify marker-trait associations for biomass yield and quality parameters that are important for biorefinery purposes.

Acknowledgments: We thank Morten Læssøe Stephensen from the University of Copenhagen and Ole Andersen, Erling and Helle Hisselholm, and other employees at Sejet Plant Breeding A/S for their help with plant harvests and the recording of phenotypic data. Britta Skov and Anja Hecht Ivø of the University of Copenhagen are acknowledged for 
their technical support with the laboratory analyses. The project was funded by Sejet Plant Breeding A/S and Innovation Fund Denmark, grant 12-132625 (Bio-Value: Value added products from biomass to J.K.S.).

\section{Compliance with Ethical Standards:}

Disclosure of potential conflicts of interest: The authors declare that they have no conflict of interest.

Research involving Human Participants and/or Animals: This article does not contain any studies with human participants or animals performed by any of the authors.

Informed consent: Not applicable.

Data availability statements: The datasets generated during and/or analysed during the current study are available from the corresponding author on reasonable request.

\section{Introduction}

Winter wheat (Triticum aestivum L.) breeding is crucial to obtain a sustainable and competitive wheat production and would benefit from the continual development of molecular-markers to select new varieties with improved growth characteristics. The green revolution and the discovery of reduced height (Rht) genes in particular have had a considerable impact on increasing grain yields while simultaneously reducing straw biomass and lodging (Gent and Kiyomoto 1997; Hedden 2003; Pearce et al. 2011; Thomas 2017). One challenge for Danish wheat breeders has been to overcome the problems of strict regulations on nitrogen fertilization in order to maintain yield with reduced fertilizer use (van Grinsven et al. 2012; Dalgaard et al. 2014), and breeding programmes have therefore focused on delivering new varieties with competitive yields at lower fertilizer application. In order to introduce greater value into their winter wheat varieties, dual-purpose varieties are of interest to breeders because they could provide food for the world's growing population along with bio-based raw materials that can replace those from fossil sources (Townsend et al. 2017). A dual-purpose wheat variety can be used for both food/feed and biorefinery purposes, thus utilizing the whole biomass and making agricultural production more sustainable.

In winter wheat, marker-trait associations (MTAs) and genetic markers have been studied intensively for grain yield (Crossa et al. 2007; Bordes et al. 2014; Ogbonnaya et al. 2017; Assanga et al. 2017), harvest index (Edae et al. 2014; Ain et al. 2015; Sukumaran et al. 2015; Qaseem et al. 2018), plant height (Liu et al. 2011; Zhang et al. 2011; Griffiths et al. 2012; Zanke et al. 2014; Bellucci et al. 2015), lodging (Verma et al. 2005; Zhang et al. 2008; Berry and Berry 2015), and STB susceptibility (Brown et al. 2015; Arraiano and Brown 2017), while few studies have been undertaken on straw biomass (Sukumaran et al. 2015; Qaseem et al. 2018) and straw quality (Bellucci et al. 2015). It is known that MTAs for grain yield are distributed throughout the genome, as also documented in a study by Crossa et al. (2007) in which only chromosome 4D showed no MTAs. Plant height and lodging are largely controlled by Rht genes, and allele $R h t-B 1 b$ and Rht-D1b in particular, located on chromosome 4B and 4D respectively, are of great importance (Würschum et al. 2015). A reduced plant height gene has been demonstrated on chromosome 2D (Rht8) (Korzun et al. 1998) and Rht alleles on 4D (Rht-D1c and Rht-D1d) and in 4B (Rht-B1c, Rht-B1d, Rht-B1e, and Rht-B1f) have also been identified (Börner et al. 1996; Pearce et al. 2011). Another study has also shown that plant height seems to be controlled by many small and major effector genes throughout the wheat genome and that many of the SNPs have an additive effect (Zanke et al. 2014). Besides being controlled by Rht-B1 and Rht-D1 genes, lodging has also been 
associated with chromosomes 1B, 1D, 2B, 2D, 3A, 6D and 7D (Verma et al. 2005; Zhang et al. 2008). The harvest index has been increased by the introduction of the semi-dwarfing genes and MTAs for harvest index have been found on chromosomes 1B, 1D, 3B, 3D, 5A, 6B and 7A (Edae et al. 2014; Ain et al. 2015; Sukumaran et al. 2015; Qaseem et al. 2018). Identification of markers for Septoria tritici blotch (STB), a wheat disease caused by the fungus Mycosphaerella graminicola (anamorph Septoria tritici) (Brading et al. 2002), is becoming increasingly important due to the development of resistance to fungicides. A recent search revealed MTAs for STB on all chromosomes in the A genome except for 1A, and in genome B and genome D except for 4D (Brown et al. 2015; Arraiano and Brown 2017; Vagndorf et al. 2017). In addition to identifying markers for the above mentioned priority traits, it will also be relevant to include markers for straw yield and quality. Wheat straw is rich in carbohydrates (cellulose, hemicellulose and lignocellulose) and their bioconversion into ethanol ( $2^{\text {nd }}$ generation biofuel) rather than using the starchy grains is important for obtaining a sustainable biofuel production. In the present study, straw quality is defined by its ability to release monosaccharides (glucose, xylose and arabinose) from straw for fermentation or conversion into biomaterials after enzymatic saccharification.

True MTAs identified for biomass yield and quality are extremely rare, while the degree of heritability or genetic variation has been analyzed in several studies. Recently, Jørgensen et al. (2018) has shown that straw and total biomass yield in a panel of fourteen modern elite cultivars varies significantly. Other studies have identified MTAs on chromosomes 3B, 3D, 5A, 6A and 7A (Sukumaran et al. 2015; Qaseem et al. 2018). However, when looking at monosaccharide release from wheat straw after enzymatic hydrolysis, Jørgensen et al. (2018) found no genetic variation between the 14 varieties. In contrast, a study by Lindedam et al. (2014) reports genetic variation in sugar release after enzymatic saccharification of 20 modern and old varieties. Genetic variation has also been further detected in GWAS of an historical Scandinavian winter wheat collection, where a weak MTA for glucose release was identified on chromosome 1B (Bellucci et al. 2015).

Many of the previous studies on wheat have used breeding programmes specifically designed for the traits analyzed in the MTA analysis. The use of active breeding nurseries to identify genetic markers is more challenging than using a mix of modern elite lines, old varieties and landraces. The lines used in active breeding nurseries are likely to have chromosomal regions that are highly inbred for desirable traits, and the segregation of alleles is not completely random. This might make it more difficult to identify known QTLs for cross referencing in the association study such as reduced height genes.

This study aimed to identify MTAs in an active wheat breeding nursery, rather than in a designed population, as this is what breeding companies use on a daily basis. The study therefore intended to identify GWAS algorithms that are able to capture MTAs in material from active breeding nurseries. The lines in the breeding nursery were phenotyped for a large number of agronomic traits that are important in the development of dual-purpose varieties. Three different GWAS algorithms (CMLM, SUPER and FarmCPU) were tested for identification of MTAs using 190 winter wheat lines, grown over three years using the 15k SNP array from TraitGenetics. It addressed the biometric challenge of an active breeding nursery, embracing a relatively small number of lines and with $50 \%$ of the lines removed and replaced with new lines every year. Finally, there is a discussion about the ability to identify markers for biomass yield and quality and about limitations in the breeding of dual-purpose varieties. 


\section{Growth conditions for plant material}

110 A collection of hexaploid winter wheat lines and varieties (Triticum aestivum) was grown in a field trial at Sejet Plant Breeding (Horsens, Denmark) for three years from 2013 to 2016. The experimental layout of the active breeding nursery was a randomized alpha block design with rotation, such that 50 new lines were introduced each year and 50 lines from the previous year were excluded, increasing the final number of lines grown partly over three years to 194. The yearly block design included 94 lines and two check cultivars, giving 96 plots (size $8.1 \mathrm{~m}^{2}$ ) per block. Four blocks (replicates), consisting of 12 columns and 8 rows were included in the yearly design, in which each column defined a mini-block resulting in 12 mini-blocks per block. Phenotypic data were available from four blocks in 2013/14 (with the exception of data for straw yield and quality traits which were only available from two blocks) and three blocks each for 2014/15 and 2015/16. The soil type was JB6, referring to a heavy clayey soil. The experimental site was fertilized following the yearly recommendations of the Danish Agricultural Agency (lbst.dk). Plant protection against pathogens followed the guidelines of The Danish Agricultural Advisory Service.

\section{Agronomic traits}

Traits important for yield and quality of grain and straw were obtained for all three years and included grain yield, plant height, lodging, foliar susceptibility to STB (ZymoSTB), straw yield, and sugar release after enzymatic hydrolysis of the straw.

Plant height was recorded as the length of the straw without the ear at full canopy height and the majority of lines in the breeding nursery were semi-draws either with respect to Rht-B1b or Rht-D1b. Both plant height and STB susceptibility were assessed in the field once at full height, while lodging was assessed at three different time points. The first assessment of lodging coincided with ear emergence, while the timing of the middle assessment depended on the actual intensity of precipitation events. The final assessment of lodging was at full maturity, just before harvest. The three time points when lodging was assessed are referred to as early, medium and late-growth stage. Following standard procedures by the breeders, lodging and STB susceptibility were scored on a scale from 0 to 9 , with 0 characterizing plots with no lodging or plots with no STB infection.

The straw and grain material was harvested using an experimental combine harvester providing grain (whole plot) and a straw sample representative for the whole plot. The weights of the grain, the straw sample and straw from the entire plot were recorded directly in the field. The straw samples were later re-measured after they had been dried (for two days at $60^{\circ} \mathrm{C}$ for straw) to calculate water loss. The grain yield of each plot was calculated assuming a water content of $15 \%$ (equations for grain yield, straw yield and harvest index are provided in supplementary material eq. 1 to 4 ).

\section{Pretreatment, enzymatic saccharification and quantification of sugars}

To assess the sugar release from straw after enzymatic saccharification, straw material was cut into 4 mm fragments and further milled into fine powder by ball milling using a robotic system (Labman Automation Ltd, Stokesley, North Yorkshire, UK; see also Studer et al. 2010; Selig et al. 2010; Zhang et al. 2014). The pretreatment was conducted using a high-throughput system with 96-well plates as described by Zhang et al. (2014). In brief, 27.0-30.0 mg of the sample was pretreated in triplicates in each 96-well plate. For each plate, three control samples and 3 blanks were included. To 
each well, $422 \mu \mathrm{l}$ of $50 \mathrm{mM}$ citrate buffer $\mathrm{pH} 5.0$ was added and the plates were heated to $190{ }^{\circ} \mathrm{C}$ for 10 minutes. The enzymatic hydrolysis was performed in the plate using Cellic CTec2 (a mixture of aggressive cellulases, $\beta$-glucosidases and hemicellulase) with an enzyme loading of 5 FPU/g DM sample (1g enzyme to $16 \mathrm{~g}$ buffer), provided by Novozymes. Hydrolysis was performed at $50^{\circ} \mathrm{C}$ for 72 hours during which time the samples were shaken at 450-600 rpm. The hydrolysis was terminated by heat-inactivation at $90^{\circ} \mathrm{C}$ for 30 minutes. The plates were centrifuged (rotor 11240, Sigma 3-18K) at $1100 \mathrm{~g}$ for 10 minutes and the supernatant filtered through a microtiter filter $(0.45 \mu \mathrm{m}$ GHP membrane, product no. 8084, Pall Corporation AS) mounted in a microtiter plate (product no. 82.1581, Hounisen AS). The samples were stored at $4{ }^{\circ} \mathrm{C}$ for a maximum of three days before analysis. For the analysis, $5 \mu \mathrm{l}$ of the sample was transferred to a new microtiter plate and diluted with $245 \mu \mathrm{l} 50 \mathrm{mg} / \mathrm{L}$ fucose solution. Quantification of glucose, xylose and arabinose was performed using a Dionex IC5000 HPAEC-PAD under isocratic conditions (20mM NaOH) with a Dionex CarboPac ${ }^{\mathrm{TM}}$ PA1 (2 x 50mm) guard column and a Dionex CarboPac ${ }^{\mathrm{TM}}$ PA1 (2 x 250mm) analytic column.

The following equation was used to convert the results obtained by sugar analysis into milligram monosaccharide released per gram dry matter (mg/g DM):

$$
\text { Monosaccharide }=\frac{C \times V \times 1000}{D M \times k}
$$

where $C$ is the concentration of monosaccharide released $(\mathrm{g} / \mathrm{L}), V$ is the total liquid volume $\left(0.472 \times 10^{-3} \mathrm{~L}\right)$ added to each well, DM is the quantity of DM analysed (g) and k is the hydration factor (1.111 for glucose and 1.136 for arabinose and xylose). Inclusion of the hydration factor accounted for hydration caused by depolymerisation.

The sugar release data were adjusted for plate-to-plate variation and differences in the assay performance between years using standards. The standards consisted of either a mixture of samples from the 2014 harvest included in each plate or of samples analysed in both 2015 and in 2017 (control samples). For normalization of plate-to-plate variation, a multiplication factor was calculated by the following equation:

$f_{m, p}=\frac{\overline{X_{C, T o t a l}}}{\overline{X_{C, P l a t e}}}$

where $\overline{X_{C, \text { Plate }}}$ denotes the mean of standards for each plate and $\overline{X_{C, \text { Total }}}$ denotes the mean of standards for all plates. This enabled the creation of a multiplication factor for each plate, which was multiplied with each sugar measurement on that plate to adjust for plate-to-plate variation. Saccharification of samples harvested in 2014 was performed in 2015 , while samples harvested in 2015 and 2016 were analysed together in 2017. To normalize for variation between the rounds of saccharification, mainly associated with enzyme batch performance, 10 samples from the 2014 harvest were randomly chosen and included in the saccharification performed in 2017 (control samples). The difference in mean sugar release for the 10 control samples analysed in 2015 and in 2017 was then used to adjust the saccharification conducted in 2015.

Correlations between monosaccharide release after enzymatic hydrolysis and straw yield was calculated in $\mathrm{R}$ using the pairs.panels function applying Pearson correlations. 


\section{Genotyping}

DNA extracted from the leaf material of 194 lines were genotyped by TraitGenetics GmbH

(http://www.traitgenetics.de) using the Illumina iScan 15K SNP array (Akhunov et al. 2009). Data were obtained for 13006 SNPs and 5552 markers remained after a QC check in which markers with more than 10 \% missing data, more than $5 \%$ heterozygosity, or a minor allele frequency below $5 \%$ were removed (also removing monomorphic markers). The final number of cultivars was reduced to 190, as four lines from year 2016 were removed, one failed to produce DNA for SNP genotyping, and the other three lines showed more than $5 \%$ heterozygosity. SNP data were converted to numeric format using the numeric conversion option in GAPIT, with the major allele, minor allele and heterozygotes given the numbers 1, 3 and 2 respectively. These were then converted to 0,2 and 1 , which is the numeric format used in GAPIT. The missing values were then imputed using the KNN function in R (knncatimpute), which is a method that imputes the missing values based on $\mathrm{k}$ nearest neighbours $(\mathrm{k}=3)$ (Troyanskaya et al. 2001). The numeric formatted SNP data with imputed markers were used for GWAS in R. R version 3.2.1 was used for all calculations, statistical tests and plots and Inkscape 0.92 was used for figure editing.

\section{Estimated trait means and genetic variation}

Phenotypic trait data were analysed for outliers and modelled through mixed linear models using the R-package ASreml-R (VSN International, Hemel Hempstead, UK). The modelling removed variations caused by environmental sources, such as block and year differences, or variations caused by technical errors during laboratory analyses such as pipetting uncertainties and plate-to-plate variation. For a given variable $Y$, corresponding to a trait value, the following model was applied:

$$
Y=X \beta+Z u+\epsilon
$$

Equation 6 consists of a vector of random effects $u$, a vector of fixed effects $\beta$, two design matrices $X$ and $Z$ with the residual $\epsilon$ denoting the random error. The design matrices associate trait observations with the appropriate fixed effects and random effects. The vector of random effects $u$ and fixed effects $\beta$ are defined in equations 7 and 8 respectively, and consists of a $\mathrm{p} \times 1$ and $\mathrm{q} \times 1$ vectors:

$$
\begin{aligned}
& u=A_{i}+B_{k i}+b_{m i}+R_{j i}+C_{l i}+G x E_{n i} \\
& u=A_{i}+B_{k i}+b_{m i}+R_{j i}+C_{l i}+G x E_{n i}+P_{o}+P_{p} \\
& \beta=\mu+G_{j}
\end{aligned}
$$

In equation $8, \mu$ defines the overall mean (eq. 7 and $7.1 \mu=0$ ). The other parameters in equation 7 are the explanatory variables that defines the random effect of year variation $\left(A_{i}\right.$, where $\left.i=2013-2015\right)$, field variation consisting of block $\left(B_{k i}, k=1-4\right)$, mini-block $\left(b_{m i}, m=1-48\right)$, row $\left(R_{j i}, j=1-48\right)$, and column $\left(C_{l i}, l=1-12\right)$, and the genotype by year/location effect $\left(G \times E_{n i}, n=1-190\right)$. The explanatory variable of equation 8 defines the fixed effect of the genotypic variation $\left(G_{n}, n=1-190\right)$. Equation 7.1 shows the random effects included for sugar release data, where plate number $\left(P_{o}, o=1-30\right)$ and plate position $\left(P_{p}, p=1-96\right)$ are also included. 
Mixed linear models were used to estimate means (best linear unbiased prediction, BLUPs) such that the most accurate phenotypic estimate was used in GWAS. The best predicted trait values were used to show the distribution of the data (Fig. S1). The mixed linear model was modified for estimation of broad sense heritability by including genotypic variation as a random effect rather than a fixed effect. Broad sense heritability is defined here as the variation estimate of an individual measurement that was based on a random replicate, a random variety, and a random location in the field (Visscher et al. 2008; Leplat et al. 2016),

$$
H^{2}=\frac{\sigma_{G}^{2}}{\sigma_{P}^{2}}=\frac{\sigma_{G}^{2}}{\sigma_{G}^{2}+\sigma_{G x E}^{2}+\sigma_{e}^{2}}
$$

where the variance terms for phenotypic variance $\left(\sigma_{P}^{2}\right)$ consisted of variance components of the explanatory variables of the random effects of genotype, genotype by environment $\left(\sigma_{G x E}^{2}\right)$ and the residual variance ( $\sigma_{e}^{2}$, the error term). The repeatability $\left(R^{2}\right)$ for cultivar-means (the heritability of variety means (Leplat et al. 2016)), as applied in Sukumaran et al. (2015) , was calculated using

$$
R^{2}=\frac{\sigma_{G}^{2}}{\sigma_{G}^{2}+\frac{\sigma_{G X E}^{2}}{Y}+\frac{\sigma_{e}^{2}}{Y R}}
$$

where $\sigma_{G}^{2}$ denotes the genetic variation, $\sigma_{G x E}^{2}$ the genotype by year variation and $\sigma_{e}^{2}$ the residual variation. $Y$ is the number of years and $R$ is the number of replicates.

\section{Genome-wide association analysis}

Three different mixed linear model algorithms, all available in R, were used to run GWAS in order to identify the association between SNP markers and traits. The first and second were the mixed linear models CMLM (Compressed Mixed Linear Model; (Zhang et al. 2010)) and SUPER (Settlement of MLM Under Progressively Exclusive Relationship; (Wang et al. 2014)), supported by GAPIT 2.0 (Genome Association and Prediction Integrated Tool) (Lipka et al. 2012). The third model was FarmCPU (Fixed and random model circulating probability unification; (Liu et al. 2016)) supported by FarmCPU (now included in GAPIT 3.0). GAPIT has been widely used to identify QTLs and the newer integrated algorithm SUPER is designed to increase the power compared to CMLM (Wang et al. 2014). However, these methods suffer from accepting false positives. Normally, the kinship matrix (K-matrix) and inclusion of principal components (PCs) will control for false positives, but in return it also weakens the power such that more false negatives are obtained. FarmCPU improves the control of false positives and false negatives caused by the confounding problem between PC’s or K-matrix and test markers by controlling the inflation of p-values (Liu et al. 2016).

All three methods control for false positives by fitting a Q-matrix (population structure or PCs) and a K-matrix (family relatedness) as covariates, but only SUPER and FarmCPU include pseudo quantitative trait nucleotides (pseudo QTNs) as covariates as well. A pseudo-QTN defines a SNP that is associated with the trait.

In CMLM, the K-matrix and Q-matrix are constant irrespective of the trait analysed and the K-matrix is based on groups of individuals. CMLM has three different algorithms for generating the K-matrix, VanRaden, Emma, and Loiselle (Zhang et al. 2010), which were all tested in the present study. 
In SUPER and FarmCPU, the kinship matrix differs from trait to trait as only SNPs affecting the trait are included as pseudo QTNs for deriving the kinship matrix. The SUPER algorithm is based on the FaST-LMM-Select model (factored spectrally transformed linear mixed models) which is a mixed effect model that incorporates a map of markers when estimating pseudo QTNs (Wang et al. 2014).

The FarmCPU algorithm combines the models FaST-LMM-select model (testing genetic markers one at a time) and the Multi-locus Mixed model (MLMM) (testing multiple markers simultaneously) in order to remove any confounding between test markers and $\mathrm{K}$ and Q matrices. The FarmCPU algorithm is therefore based on a fixed effect model and a random effect model, which are run separately and repeatedly and affect one other (Liu et al. 2016).

Fixed effect model:

$$
y_{i}=S_{i 1} E_{1}+\cdots+S_{i t} E_{t}+s_{i j} e_{j}+\epsilon_{i}
$$

Random effect model:

$$
y_{i}=u_{i}+\epsilon_{i}
$$

251

In both models, $y_{i}$ defines the trait value of the $i^{\text {th }}$ wheat cultivar and $\epsilon_{i}$ corresponds to the residual $\sim N\left(0, \sigma_{\epsilon}^{2}\right)$. In the fixed effect model $S_{i 1} \ldots S_{i t}$ and $E_{1} \ldots E_{t}$ gives the $i^{\text {th }}$ and the $t^{\text {th }}$ pseudo QTNs genotype and effect respectively, where the first defines the covariates that are zero at the beginning. $s_{i j}$ corresponds to the genotype of the $i^{\text {th }}$ wheat cultivar and the $j^{\text {th }}$ SNP marker, while $e_{j}$ is the effect of the $j^{\text {th }}$ SNP marker. In the random effect model, $u_{i}$ defines the total genetic effect of the $i^{\text {th }}$ wheat cultivar, where the variance and covariance matrix is given by $G=2 K \sigma_{a}^{2}$. $K$ is the kinship matrix created based on pseudo QTNs and $\sigma_{a}^{2}$ is the genetic variance (Liu et al. 2016).

The fixed effect model included pseudo-QTNs as covariates and test markers that were tested one at a time. The association between test markers and the trait was validated by the random effect model with K derived from pseudoQTNs. The two models were run repeatedly until no more pseudo-QTNs were added to the fixed effect model. The pvalue of the marker that corresponded to the pseudo-QTN was substituted by the most significant p-value of the pseudoQTN.

The SUPER method, which consists of the random model, is used to optimize pseudo-QTNs, incorporating the genetic map information and test statistics from the fixed effect model. The individual's genetic effect, including variance and covariance matrix, was used as a random effect. After the evaluation of pseudo-QTNs, the pseudo-QTNs are inserted back into the fixed effect model and this continues until no more pseudo-QTNs are added to the model (Liu et al. 2016).

Irrespective of the GWAS algorithm applied, SNPs were considered to be significantly associated with a trait at a pvalue below $9.00576 \times 10^{-6}\left(p=\frac{0.05}{n o . S N P S}\right)$, corresponding to a $-\log 10(p)$ above 5.05 . If no markers exceeded this threshold, weak associations represented by SNP markers that had a p-value below 0.001 , corresponding to a -log10(p) above 3, were analysed instead. 
Validation of genotyping

The filtration of the SNP markers reduced the 15k SNP array to 5552 markers. These were relatively evenly distributed across the 21 chromosomes (Table 1). The SNP frequency was significantly lower on all chromosomes in the D genome compared to the other two genomes, as was expected due to the later introduction of the D genome in hexaploid wheat. The coverage was also lower on chromosome 4 in all genomes. For chromosomes 4B and 4D this might be due to less recombination because the majority of lines in this study were semi-dwarfs (either Rht-B1b or Rht-D1b) and therefore more inbred at these chromosomes. Consequently, detection of rht markers might be more difficult compared to a study that also includes landraces.

Table 1 Distribution of SNPs across the 21 wheat chromosomes

\begin{tabular}{ccccccccc} 
Genome $\backslash$ Chromosome & $\mathbf{1}$ & $\mathbf{2}$ & $\mathbf{3}$ & $\mathbf{4}$ & $\mathbf{5}$ & $\mathbf{6}$ & $\mathbf{7}$ & Total \\
\hline A & 308 & 336 & 284 & 161 & 375 & 372 & 394 & 2230 \\
B & 389 & 407 & 455 & 224 & 463 & 378 & 293 & 2609 \\
D & 141 & 129 & 86 & 38 & 108 & 124 & 85 & 711 \\
\hline
\end{tabular}

*586 additional SNP markers were unmapped and not assigned to a chromosome

The SNP markers were used to generate a kinship matrix and principal components to control for family relatedness and population structure, respectively. The kinship algorithm was consistently included in the mixed linear models used to run GWAS. The number of principal components included in the model depended on whether they improved the QQplot (quantile-quantile) and was highly dependent on the GWAS algorithm used (GAPIT-CMLM, GAPIT-SUPER, or FarmCPU). The principal components were evenly distributed and did not indicate a strong population structure (Fig. S2). Three different kinship matrices (van Raden, Emma, and Loiselle) were tested in GAPIT and all gave equally good kinship matrices that did not change the effect on the QQ-plots (Fig. 1 and S3). All three K-matrices revealed that there was no strong genetic resemblance between the lines, as illustrated by the bright white to orange colours in Figure 1a-c. The QQ-plots followed the diagonal line and did not indicate the presence of population structure (Fig. 1d-f; results shown for straw yield only, see Fig. S3 for grain yield and plant height). Based on the QQ-plot comparison (Fig.1 and S3), it is apparent that the Emma and Van Raden algorithms performed equally well, while the Loiselle algorithm experienced more noise indicating that Loiselle corrected less well for population structure (Fig. S3). Here the kinship matrix conducted with the Emma algorithm, which also happens to be the most recent kinship algorithm in GAPIT, was chosen as the kinship algorithm for all traits, but the Van Raden algorithm would have been equally good to apply. For the plot of principal components and the heterozygosity of the lines see Figure S2.

Fig. 1 Comparison of kinship matrices (a, b, and c) and the QQ-plots for straw yield (d, e, and f) between the three algorithms van Raden, Loiselle, and Emma: a) kinship matrix and d) QQ-plot when applying the van Raden algorithm, b) and e) when applying the Loiselle algorithm and c) and f) when applying the Emma algorithm. The K-matrix illustrates the genetic resemblance between the 190 lines as shown by the heat map and the dendrograms (each axis). The diagonal line represents inbred lines as is revealed by a dark orange colour. The darker colour, the more inbred the lines. Straw yield was chosen as an example of the similarity between the QQ-plots. The QQ-plot shows the expected $\log 10(p)$ values plotted against the observed -log10(p) values. The grey shadow resembles the 95\%-confidence interval 
Evaluating GWAS algorithms

Three different GWAS algorithms (GAPIT-CMLM, GAPIT-Super and FarmCPU) were used to analyse the data in order to test which algorithm provided the best QQ-plot combined with the lowest p-values. The GAPIT-Super algorithm were not able to correct properly for population structure for all traits as the p-values seemed to be inflated (above the diagonal) and the QQ-plot showed more noise (Fig. S4.b). The fitness of the lines in the QQ-plots for FarmCPU and GAPIT-CMLM were in general comparable, as they followed the diagonal well, but for most traits FarmCPU decreased the p-values compared to GAPIT-CMLM (Fig.S4.a-c with plant height as an example). There were also cases where FarmCPU failed to improve and even slightly reduced the quality of the QQ-plot compared to GAPITCMLM, as illustrated for straw yield and grain yield in Figure S4a-c. FarmCPU have a tendency of overcorrecting their models, with the straight line a bit below the diagonal, but the algorithm experience a minimum of noise as the line are straight (QQ-plot for plant height Fig.S4.a). Besides this FarmCPU had the ability to detect marker-trait associations with lower p-values for the majority of traits (plant height is shown as an example in Fig.S4) compared to the two other algorithms. As all algorithms failed to identify MTAs for grain yield and straw yield and the FarmCPU algorithm succeeded in identification of MTAs for the majority of the other traits, the FarmCPU algorithm was used as the GWAS algorithm for all traits.

The effect of including principal components

The advantage of including principal components (PCs) in GWAS was tested in the GAPIT software using Bayesian information criterion (BIC) values. For all traits in this study, exclusion of PCs gave the highest BIC values, therefore zero PCs were applied in GAPIT. Since the same test could not be performed in FarmCPU, all combinations of PCs were included in GWAS with the number of PCs included for each trait being based on improvements in the QQ-plot. If the QQ-plot was unchanged by including more PCs, then the model with the least number of PCs was chosen. The number of PCs included in FarmCPU therefore differed between traits and the inclusion or exclusion of PCs had a considerable effect on correcting for the population structure.

Modelling agronomic and biorefinery traits

Based on a visual inspection of the distribution of the predicted phenotypic means all traits with exception of early and intermediate lodging seemed to be normal distributed (Fig. S1), however only means of plant height, grain yield, STB and harvest index passed the Shapiro-Wilk normality test $(\mathrm{p}>0.001)$ while the remaining traits failed to pass it (See table S1). Logarithmic transformation of data did only improve normality for straw yield, but not enough for it to become normal distributed. Early lodging had a skewed distribution due to the high number of zeros, i.e. breeding lines that did not show any lodging. Residual-plots (normal probability plots and residuals vs. fitted value plots) showed that residuals were normally distributed (data not shown). Table 2 lists the summary information (mean, range and coefficient of variance) for the phenotypic data before and after being modelled through mixed linear models to predict estimates for the phenotypic trait values for each breeding line.

Table 2 Range of agronomic and biorefinery traits

\begin{tabular}{lllllll}
\hline Phenotype & \multicolumn{2}{c}{ Mean } & \multicolumn{2}{c}{ Range $^{\text {CV [\%] }}$} & \multicolumn{2}{c}{ Raw $^{\text {Estimate }}$} \\
\cline { 2 - 7 } & Raw & Estimate $^{\mathbf{a}}$ & Raw & Estimate $^{\mathbf{a}}$ & Raw & 15 \\
\hline Straw yield [t/ha] & 7.7 & 7.5 & $3.6-18.6$ & $3.7-12.1$ & 28 & 7 \\
Plant height [cm] & 85 & 85 & $63-110$ & $70-99$ & 11 & 7 \\
Grain yield [t/ha] & 9.0 & 9.0 & $6.9-10.9$ & $7.2-10.1$ & 5 \\
\hline
\end{tabular}




\begin{tabular}{lllllll}
\hline Harvest index [\%] & 55 & 55 & $31-70$ & $43-70$ & 11 & 7 \\
Early lodging [score] & 1 & 0 & $0-7$ & $-1-3$ & 845 & 554 \\
Intermediate lodging ${ }^{\text {[ }}$ score] & 3 & 3 & $0-9$ & $-3-11$ & 106 & 62 \\
Late lodging [score] & 3 & 3 & $0-9$ & $-3-9$ & 124 & 82 \\
STB [score] & 5 & 5 & $2-7$ & $4-6$ & 17 & 13 \\
Arabinose ${ }^{\mathrm{b}}$ [mg/g DM] & 11.1 & 11.1 & $6.1-19.1$ & $9.6-12.6$ & 16 & 5 \\
Xylose [mg/g DM] & 129.3 & 130.0 & $61.4-201.0$ & $112.8-151.6$ & 13 & 5 \\
Glucose [mg/g DM] & 184.1 & 184.1 & $103.7-287.5$ & $158.3-211.4$ & 14 & 5 \\
\hline
\end{tabular}

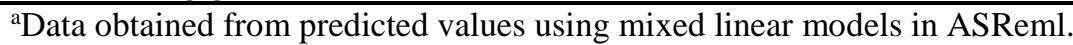

'Data obtained from two years data compared to three years' data as for the other traits

The coefficient of variance (CV) defines the quality of the data and it was acceptable (below 30\%) for the majority of traits. However, CV was generally high for lodging, which might be due to the relatively high number of cases of zero (absence of lodging), variation between years and the absence of normally distribute data.

Table 3 shows the heritability for each phenotype and the components of genetic variance, the genotype by year ( $\mathrm{G} \times \mathrm{E}$ ) variance and the stochastic and measurement error variance. The repeatability $\left(\mathrm{R}^{2}\right)$ of each trait, taking the number of locations, years and replicates into account, is also given in Table 3.

Heritability was relatively low for many traits including sugar release, early stage lodging, harvest index and straw yield, ranging between 0.02 and 0.15 (Table 3). The genotype by environment $(\mathrm{G} \times \mathrm{E})$ variance $\left(\sigma_{G x E}^{2}\right)$, defining the genotype by year variance, was also comparable or greater than the genetic variance for most of these traits, with the exception of harvest index. The $\mathrm{G} \times \mathrm{E}$ variance for sugar release traits was similar to the genetic variation $\left(\sigma_{G}^{2}\right)$, while the error variance $\left(\sigma_{e}^{2}\right)$ had the grates effect with respect to reducing heritability. This was illustrated by the error variance being 15x, 27x, and 50x greater than the genetic variation for xylose, arabinose and glucose, respectively. The major contributors to the error variance for monosaccharide release was believed to be coursed by analytical and experimental variances, which could indicate that the low heritability was caused by experimental noise and that proper noise correction or other methods might be able to uncover more genetic variation. $H^{2}$ was based on all replicates without adjustment for the number of replicates and years, as was done for $R^{2}$; this difference decreased the error variance and the $\mathrm{G} \times \mathrm{E}$ variance and made the $R^{2}$ estimates higher. Heritability for STB susceptibility, plant height, grain yield and the later stages of lodging ranged between 0.35 and 0.74 , while the repeatability for the same traits was between 0.67 and 0.94 (Table 3).

Table 3 Variance estimates for the different traits including broad sense heritability and repeatability

\begin{tabular}{|c|c|c|c|c|c|c|c|c|c|}
\hline Phenotype & $\sigma_{G}^{2}$ & $\sigma_{G x E}^{2}$ & $\sigma_{e}^{2}$ & $\sigma_{\text {Total }}^{2}$ & $\% \mathrm{G}$ & $\% \mathrm{G} \times \mathrm{E}$ & $\%$ e & $H^{2 b}$ & $R^{2}$ \\
\hline Straw yield $^{\text {a }}$ & 0.006 & 0.01 & 0.03 & 0.046 & 13.04 & 21.78 & 65.22 & 0.15 & 0.47 \\
\hline Plant height & 33.65 & 3.62 & 8.00 & 45.27 & 74.33 & 8.00 & 17.67 & 0.74 & 0.94 \\
\hline Grain yield & 9.48 & 10.97 & 4.90 & 25.35 & 37.40 & 43.28 & 19.33 & 0.37 & 0.69 \\
\hline Harvest index & 2.59 & 8.87 & 19.9 & 31.36 & 8.26 & 28.28 & 63.46 & 0.08 & 0.33 \\
\hline Early lodging & 0.01 & 0.05 & 0.16 & 0.22 & 4.55 & 22.73 & 72.73 & 0.05 & 0.24 \\
\hline $\begin{array}{l}\text { Intermediate } \\
\text { lodging }\end{array}$ & 1.99 & 2.41 & 1.19 & 5.59 & 35.60 & 43.11 & 21.29 & 0.35 & 0.67 \\
\hline Late lodging & 2.30 & 2.87 & 1.08 & 6.25 & 36.80 & 45.92 & 17.28 & 0.58 & 0.68 \\
\hline STB & 0.27 & 0.09 & 0.17 & 0.53 & 50.94 & 16.98 & 32.08 & 0.51 & 0.84 \\
\hline Arabinose & 0.06 & 0.08 & 1.64 & 1.78 & 3.37 & 4.49 & 92.13 & 0.04 & 0.17 \\
\hline Xylose & 11.53 & 8.26 & 169.39 & 189.18 & 6.09 & 4.37 & 89.54 & 0.06 & 0.35 \\
\hline Glucose & 8.81 & 21.56 & 441.91 & 472.28 & 1.87 & 4.57 & 93.57 & 0.02 & 0.14 \\
\hline
\end{tabular}


${ }^{\text {a }} \log$ transformed data

${ }^{\mathrm{b}}$ Calculations were conducted before the variance components were rounded to two decimals $\sigma_{G}^{2}$ defines the genetic variance, $\sigma_{G x E}^{2}$ the genotype by year variance, $\sigma_{e}^{2}$ the error variance, $\sigma_{\text {Total }}^{2}$ the sum of the three variance components (total variance), $H^{2}$ is broad sense heritability and $R^{2}$ is repeatability. $\% \mathrm{G}, \% \mathrm{G} \times \mathrm{E}$ and $\%$ e defines how much of the total variance the specific variance components accounts for in percentage.

GWAS results

\section{Lodging and plant height}

The agronomic trait lodging was scored three times, viz. twice during development (early and intermediate), and once just before harvest (late) to reveal if lodging during plant development and before harvest was controlled by both common and different genes, with the priority given to genes for late lodging. Fig. 2 shows the Manhattan plots from GWAS run by FarmCPU, revealing the SNPs and their chromosomal location that were significantly associated with lodging and plant height.

For early lodging, significantly associated SNPs were located on chromosomes 3B, 5B, 6A, 7A, and 7D with two markers on 5B being the most significant $(-\log 10(p)$ at 18.3 and 19.2 respectively) (Table 4). No significantly associated markers were identified for intermediate stage lodging, however, there was a weak association for markers on chromosomes 1B, 4B, 5B, and 7A which had a $-\log 10(p)$ above 3 , to be specific 3.02, 3.41, 3.11 and 3.13 respectively (Fig. 2). MTAs were identified for late stage lodging on chromosome 1B, 2A, 3D, 4B, 5B and 6D with $\log 10(p)$ values between 5.1 and 8.6 (Fig. 2 and Table 4). Five MTAs were identified for plant height; one on chromosomes 1B, 6B, and 7B and two on chromosome 4B (Fig. 2). Their $-\log (p)$ scores ranged from 5.3 to 6.6 (Table 4).

Four markers for early stage lodging and one marker for late stage lodging had a minor allele frequency (MAF) close to the limit of 0.05 , which for early stage lodging could be explained by the greater frequency of absence of lodging at this stage. Beside this, the MAFs were between 0.15-0.16, 0.15-0.47, and 0.16-0.48 for early stage lodging, late stage lodging and plant height respectively (Table 4).

Boxplots in Figure 3 and the SNP effect in Table 4 illustrate the SNP effect for lodging and plant height. The SNP effect can be described as the difference between the two allelic versions. For early stage lodging, the SNP effect corresponding to reducing early stage lodging by between 0.1 and 2.2 points (on a scale from 0-9) depended on the SNP. A reduction of 2.2 points could be of great importance for minimizing early lodging. The markers identified for late stage lodging could decrease lodging between 1.2 and 1.9 points, which corresponds to a reduction of mean lodging by approximately 36\%. The largest SNP effects were for the markers BS00009777_51 and Ra_c31466_694 on chromosomes 5B and 7A respectively (2.18 and 2.19, on a scale from 0 to 9) for early lodging while marker GENE2712_130 and Ku_c18920_414 on chromosome 3D and 1B had the greatest SNP effect for late lodging (Table 4). The markers on 5B and 1B were also the second most significant markers for early and late lodging respectively. The most significant marker for early lodging (wsnp_Ex_c9362_15546626, 5B) only had a minor SNP effect of 0.1 while the marker for late lodging (Tdurum_contig91519_224, 2A) had a more pronounced SNP effect of 1.55 (Table 4). This 
could reflect the low number of lines that showed early lodging (not normally distributed data). For plant height the SNP substitution was able to decrease plant height by between 2.3 and $3.8 \mathrm{~cm}$ and the markers on $1 \mathrm{~B}$ and $4 \mathrm{~B}$ were those with the greatest SNP effect and also the most significant for plant height.

No MTAs were shared between all three lodging stages as no MTAs were observed for intermediate stage of lodging. Shared MTAs for early and late lodging were in general different, except for MTAs located in a single chromosomal region on $5 \mathrm{~B}$ (from $403.9 \mathrm{Mb}$ to $712.9 \mathrm{Mb}$ ) (Table 4). If weak associations for intermediate lodging, corresponding to markers with a $-\log 10(p)$-value above 3 , were included then there were overlapping chromosomal regions identified between the early and intermediate stage lodging (7A) and late and intermediate stage lodging (1B and 4B). The markers identified on chromosome 7A differed, but they were located in the same region (586.2Mb and 732.8Mb for early and intermediate-stage lodging, respectively). The markers identified for both intermediate (weak associations) and late stage lodging on chromosome 1B and 4B were identical, hence they could share the QTLs. The MTA on 1B for late stage lodging was also identical to the MTA identified for plant height and the MTAs on 4B for both plant height and late lodging were in the same chromosomal location (506.1-552.8 for plant height and 552.0 for lodging) (Table 4).

Fig. 2 Manhattan plots for lodging and plant height. The significance level is set at a $-\log 10(p)$ of 5.05 illustrated by the solid green line. The dotted black line defines a $-\log 10(p)$ at $3(p=0.001)$ and is used as a threshold to illustrate the chromosomes where there are some weak associations

Fig. 3 Boxplot showing the SNP effect for a) early lodging, b) late lodging, c) plant height and d) harvest index. The yaxis defines the score of lodging and the x-axis defines SNP allele 1 and SNP allele 2, corresponding to absence and presence, respectively. The two boxplots visualise the estimated means of lodging for all 190 lines and how the mean changes depending on the differences in allele1 and 2

Grain yield, harvest index, and STB

The results of the association analysis for grain yield, harvest index and STB susceptibility are illustrated in Figure 4. No significant MTAs for grain yield were detected, only weak associations at a $-\log 10(p)$ at 3 , corresponding to a pvalue at 0.001 , which for grain yield was on chromosome 1A, 1B, 2A, 3B, 5B, 7A, 7B, and 7D. The most promising marker (lowest $p$-value) was found on chromosome 7A with a $-\log 10(p)$ at 3.6, while the other markers had a $-\log 10(p)$ of between 3.0 and 3.6 (Fig. 4).

For the harvest index, two SNPs were significantly associated on chromosome 2A and 5B with $-\log 10(p)$-values of around 6 and an effect size of $2.5 \%$ and $2.1 \%$ respectively, suggesting that substituting the allele increases the harvest index by approximately $2 \%$ (Table 4).

STB susceptibility also had markers associated significantly, namely those located on chromosomes 1A, 3B, 5B, 5D, 6B, and 7A with marker Ku_c22026_1410 located on chromosome 6B being the most significant (see Table 4).The $\log 10(p)$-values ranged between 5.3 and 9.1 with an allele effect of between 0.3 and 0.6 on a scale from 0 to 9 .

Two markers for STB susceptibility (BS00012069_51 and BS00110130_51) had a minor allele frequency of around 0.08 , which was close to the threshold. For all other significant markers the MAFs were from 0.11 to 0.42 and from 
0.23 to 0.35 for STB and harvest index respectively (Table 4). None of the observed MTAs for STB and harvest index and weak associations for grain yield were identical to MTAs found for other traits.

Fig. 4 Manhattan plots for grain yield, harvest index, and STB susceptibility: The significance level is set at a $-\log 10(p)$ of 5.05 which is illustrated by the solid green line. The dotted black line defines a $-\log 10(p)$ at $3(p=0.001)$ and is used as a threshold to illustrate the chromosomes where there are some weak associations

Biorefinery traits: straw quality and yield Figure 5 shows SNPs that were significantly associated with straw yield and quality traits, while the boxplots in Figure 6 illustrates the SNP effects. MTAs were observed for all types of monosaccharides $(-\log 10(p)>5.05)$, while straw yield only showed markers that were weakly associated $(-\log 10(p)>3)$. Straw yield showed too small a variation to allow marker identification, and only weak associations were found on chromosomes 1B (Ku_c18920_414) and 4B (wsnp_Ku_c12399_20037334) with - $\log 10(p)$-values of 3.0 and 3.4 respectively. The marker identified on 1B was identical to that found for plant height, which seems logical since straw yield is greatly affected by plant height (Table 4). The marker wsnp_Ku_c12399_20037334 found on 4B was located near the telomere of the chromosome (1.3Mb) while the markers identified for plant height were located towards the other end of chromosome 4B (506.1-552.8Mb) (Table 4).

MTAs were identified for all monosaccharides despite of the observed low heritabilities. Release of glucose (mg/g DM) showed MTAs on chromosome 1B and 6A with a -log10(p)-value of 7.1 and 5.8, respectively (Fig. 5). The effect of the SNP substitution increased the glucose release by between 4.8 and $7.3 \mathrm{mg} / \mathrm{g}$ DM corresponding to the mean glucose yield being increased by approximately 2-4\%.

Four MTAs were found for xylose with a $-\log 10(p)$ of between 5.3 and 7.2. The markers were located on chromosomes 5A, 5B, 6A and 7D, with the last being the most significant and having the greatest SNP effect (Table 4). The effect of substituting the SNP increased the xylose release by between 3.2 and $5.6 \mathrm{mg} / \mathrm{g} \mathrm{DM}$, also equivalent to increasing the mean xylose yield by $2-4 \%$.

Significant associations were observed for arabinose release (mg/g DM) on chromosomes 1A, 4A, 4B, and 6A ($\log 10(p)$ at 5.1 to 7.9). Their SNP effect ranged from 0.3 to $0.7 \mathrm{mg} / \mathrm{g} \mathrm{DM}$ and was able to increase the arabinose release by approximately 3-6\% (Table 4). Arabinose release had one significant marker with a MAF of 0.05 (BS00108351_51) while all other significant markers for arabinose, xylose and glucose had MAFs of between 0.09 and 0.44 (Table 4).

There was no overlap between the MTAs for biorefinery traits and those identified for other traits, but MTAs were found on chromosome 6A for all three monosaccharides. The markers were located relatively close to one another at 597.9Mb, 524.8Mb and 429.4Mb for glucose, arabinose and xylose, respectively. Chromosome 6A therefore seemed to carry important alleles for increasing the yields of all three types of monosaccharides.

Fig. 5 Manhattan plots for straw yield and quality traits, The significance level was set at a $-\log 10(p)$ of 5.05, illustrated by the solid green line. The dotted black line defines a $-\log 10(p)$ at $3(p=0.001)$ and is used as a threshold to illustrate the chromosomes where there are some weak associations 
465

466

467

468

469

Fig. 6 Boxplot showing the SNP effect for a) STB, b) glucose, c) xylose and d) arabinose. The y-axis defines the score of harvest index, STB and plant height, while absence and presence defines SNP allele 1 and SNP allele 2, respectively. The estimated means of the different traits for all 190 lines were divided into groups depending on whether they had allele 1 or 2 and plotted as boxplots

Table 4: MTAs identified by GWAS

\begin{tabular}{|c|c|c|c|c|c|c|c|}
\hline Trait & Marker type & Chr. $^{\mathrm{a}}$ & Pos. $[\mathrm{Mb}]^{\mathrm{a}}$ & \% identity & MAF & $-\log 10(p)$ & SNP effect ${ }^{\mathrm{cc}}$ \\
\hline Early & wsnp_Ex_c9362_15546626 & $5 \mathrm{~B}$ & 693.6 & 99 & 0.16 & 19.19 & 0.10 \\
\hline \multirow[t]{5}{*}{ lodging } & BS00009777_51 & $5 \mathrm{~B}$ & 712.9 & 99 & 0.15 & 18.34 & -2.18 \\
\hline & GENE-3659_162 & $6 \mathrm{~A}$ & 101.3 & 99 & 0.06 & 9.56 & 0.40 \\
\hline & Kukri_c5789_1029 & 7D & 605.0 & 99 & 0.05 & 8.55 & 0.42 \\
\hline & wsnp_Ex_c123_244117 & 3B & 485.4 & 99 & 0.05 & 7.18 & 0.42 \\
\hline & Ra_c31466_694 & $7 \mathrm{~A}$ & 586.2 & 99 & 0.05 & 6.57 & -2.19 \\
\hline \multirow[t]{6}{*}{ Late lodging } & Tdurum_contig91519_224 & $2 \mathrm{~A}$ & 451.8 & 98 & 0.27 & 8.56 & 1.55 \\
\hline & Ku_c18920_414 & 1B & 557.4 & 99 & 0.16 & 6.86 & 1.73 \\
\hline & CAP8__c3273_136 & 4B & 552.0 & 99 & 0.47 & 6.61 & 1.20 \\
\hline & BS00082101_51 & $5 B$ & 551.7 & 99 & 0.15 & 5.63 & -1.42 \\
\hline & GENE-2712_130 & 3D & 593.2 & 98 & 0.07 & 5.12 & 1.85 \\
\hline & RAC875_rep_c105937_467 & $6 \mathrm{D}$ & 708.9 & 98 & 0.19 & 5.11 & -1.18 \\
\hline \multirow{2}{*}{$\begin{array}{l}\text { Harvest } \\
\text { index }\end{array}$} & wsnp_BE406351A_Ta_2_3 & $2 \mathrm{~A}$ & 562.6 & 98 & 0.35 & 6.80 & 2.48 \\
\hline & Tdurum_contig52447_814 & $5 B$ & 456.5 & 99 & 0.23 & 5.42 & 2.06 \\
\hline \multirow[t]{5}{*}{ Plant height } & Ku_c18920_414 & 1B & 557.4 & 99 & 0.16 & 6.59 & 3.83 \\
\hline & BS0̄0022181_51 & $4 \mathrm{~B}$ & 552.8 & 99 & 0.48 & 6.38 & -2.89 \\
\hline & wsnp_Ex_c4358_7854194 & 4B & 506.1 & 99 & 0.46 & 5.75 & -2.56 \\
\hline & Kukri_c338_109 & $6 \mathrm{~B}$ & 708.7 & 99 & 0.28 & 5.31 & -2.89 \\
\hline & Kukri_c46310_84 & $7 \mathrm{~B}$ & 1.3 & 99 & 0.41 & 5.25 & -2.27 \\
\hline \multirow[t]{6}{*}{ STB } & Ku_c22026_1410 & $6 \mathrm{~B}$ & 178.0 & 99 & 0.42 & 9.12 & -0.43 \\
\hline & BS00012069_51 & $5 \mathrm{D}$ & 561.7 & 99 & 0.08 & 8.23 & -0.58 \\
\hline & Kukri_rep_c108378_52 & $5 \mathrm{~B}$ & 583.2 & 99 & 0.21 & 6.13 & -0.34 \\
\hline & Kukri_rep_c109098_132 & $7 \mathrm{~A}$ & 4.8 & 99 & 0.12 & 5.72 & 0.40 \\
\hline & Kukri_c23958_187 & 3B & 106.3 & 99 & 0.11 & 5.65 & -0.47 \\
\hline & BS00110130_51 & $1 \mathrm{~A}$ & 535.0 & 99 & 0.08 & 5.25 & 0.43 \\
\hline \multirow{2}{*}{ Glucose } & wsnp_Ex_c18384_27213023 & 1B & 619.7 & 99 & 0.18 & 7.07 & -7.25 \\
\hline & wsnp_Ex_c2236_4189774 & $6 \mathrm{~A}$ & 597.9 & 99 & 0.44 & 5.84 & -4.81 \\
\hline \multirow[t]{4}{*}{ Xylose } & RFL_Contig3607_648 & $7 \mathrm{D}$ & 634.1 & 99 & 0.09 & 7.20 & 5.57 \\
\hline & wsnp_Ex_c18965_27868480 & $6 \mathrm{~A}$ & 429.4 & 99 & 0.44 & 6.88 & -3.58 \\
\hline & RAC875_c29488_56 & $5 \mathrm{~B}$ & 663.5 & 99 & 0.12 & 6.62 & -4.79 \\
\hline & Excalibur_c53930_53 & $5 \mathrm{~A}$ & 416.2 & 98 & 0.30 & 5.31 & 3.17 \\
\hline \multirow[t]{4}{*}{ Arabinose } & BS00108351_51 & $1 \mathrm{~A}$ & 10.0 & 99 & 0.05 & 7.94 & -0.74 \\
\hline & GENE-1756_115 & $4 \mathrm{~A}$ & 593.4 & 99 & 0.26 & 5.78 & -0.30 \\
\hline & wsnp_CAP12_c1226_628910 & $6 \mathrm{~A}$ & 524.8 & 98 & 0.44 & -0.25 & 5.35 \\
\hline & BS00084226_51 & 4B & 377.8 & 99 & 0.47 & -0.26 & 5.05 \\
\hline
\end{tabular}

470

${ }^{\mathrm{a}}$ The chromosomal position of the SNP was obtained from BLAST of the marker sequence applying the new IWGSC RefSeq v1.0 sequence available at

https://urgi.versailles.inra.fr/blast_iwgsc/?dbgroup=wheat_iwgsc_refseq_v1_chromosomes\&program=blastn and marker sequences at https://triticeaetoolbox.org/wheat/.

b \% identity between the SNP marker sequence and the wheat reference genome ; 99\% corresponds to a perfect match with $1 \%$ being the SNP.

${ }^{\mathrm{c}}$ The estimate for the SNP effect defines the effect of substituting the major allele with the minor allele. The lines are homozygote, hence twice the SNP effect, which corresponds to the effect the lines with one version of the SNP vs the other will have on trait value. The sign (-) indicates the better allele. 


\begin{tabular}{llll}
\hline trait & SNP marker & SNP effect [mg/g DM] & SNP effect [kg/ha] \\
\hline Glucose & wsnp_Ex_c18384_27213023 & -7.25 & 54.57 \\
& Wsnp_Ex_c2236_4189774 & -4.81 & 36.20 \\
\hline Xylose & RFL_Contig3607_648 & 5.57 & 41.92 \\
& WSnp_Ex_c18965_27868480 & -3.58 & 26.95 \\
& RAC875_c29488_56 & -4.79 & 36.05 \\
& Excalibur_c53930_53 & 3.17 & 23.86 \\
\hline Arabinose & BS00108351_51 & -0.74 & 5.57 \\
& GENE-1756_115 & -0.30 & 2.26 \\
& WSnp_CAP12_c1226_628910 & 5.35 & 40.27 \\
& BS00084226_51 & 5.05 & 38.01 \\
\hline
\end{tabular}

Grey markers define markers located in the same chromosomal region (chromosome 5B or 6A) as markers found for other traits.

${ }^{d}$ the unit of the SNP effect corresponds to the trait unit listed in table 2.

Bold markers define identical markers

\section{Breeding for dual-purpose wheat varieties}

The heritability for release of monosaccharides was small ranging from 0.02-0.06 (Table 3) and the expected effect size obtained were therefore also low (Table 4). There still seems to be variation in data when connecting the release of monosaccharides [mg/g DM] with the straw yield [t/ha] of the specific lines (Fig. 5, eq. S5), however, the release of monosaccharides were not correlated with straw yield (Table 5) meaning that an increase in sugar release phenotype will not necessarily be followed by an increase in straw yield. When converting the effect size of [mg/g DM] to [kg/ha] based on an average straw yield, the effect size obtained seemed more pronounced compared to SNP effect values of [mg/g DM] (Table 6, eq. S5). For glucose the SNP effect could correspond to an increase in sugar release between 36 and55 kg/ha, for xylose between 24 and $42 \mathrm{~kg} / \mathrm{ha}$ and for arabinose between 2 and $40 \mathrm{~kg} / \mathrm{ha}$. If the effect of the alleles are additive then it corresponds to an increase in total monosaccharide release by 305.7 [kg/ha]. The correlation between the three monosaccharides was highly significant (Table 5) such that an increased release of glucose also results in a higher release of xylose and arabinose (Table 5). This could indicate that there are wheat lines with structures that more easily gets solubilized and accessible for enzymatic hydrolysis and therefor developing markers targeting this trait seems feasible.

Figure 5: Histograms of the frequency of sugar release in t/ha based on estimated means for sugar release and the corresponding straw yield means of each wheat line.

Table 5: Correlation between straw yield and the three monosaccharides

\begin{tabular}{|l|l|l|l|}
\hline traits & Xylose & Arabinose & Straw yield \\
\hline Glucose & $\mathrm{p}<0.001 \mathrm{r}=0.64$ & $\mathrm{p}<0.001 \mathrm{r}=0.35$ & $\mathrm{p}=0.408 \mathrm{r}=0.06$ \\
\hline Xylose & & $\mathrm{p}<0.001 \mathrm{r}=0.34$ & $\mathrm{p}=0.042 \mathrm{r}=0.15$ \\
\hline Arabinose & & & $\mathrm{p}=0.535 \mathrm{r}=0.045$ \\
\hline
\end{tabular}

Table 6: The SNP effect for sugar release in kg/ha based on average straw yield 
MTAs for traits relevant to dual-purpose wheat

The main purpose of this study was to identify MTAs for several agronomic traits, and especially for traits that impact the yield and quality of mature straw biomass, in order to facilitate the breeding of dual-purpose varieties. Many of the standard agronomic traits, including plant height, harvest index, lodging, and STB susceptibility, affect the yield and quality of straw biomass. Lodging results in greater loss of straw biomass and the quality of the straw may deteriorate due to degradation by microbes. The STB disease is also thought to reduce straw quality (Brading et al. 2002). The yield of straw biomass is related to plant height and harvest index, where breeding for more straw biomass will increase the height or density and decrease the harvest index. Grain yield was included in the association analysis because it will always be the main breeding objective.

\section{Lodging and plant height}

The MTAs observed for plant height were all located in chromosomes previously associated with plant height (Liu et al. 2011; Zhang et al. 2011; Griffiths et al. 2012; Zanke et al. 2014) however, the specific markers were not identical even though they mapped to the same chromosomal regions. The markers on chromosome 4B (RAC875_c1357_860 and RFL_Contig5341_816), reported by Zanke et al. (2014), were located at approximately the same locus (609.0 and 645.3Mb respectively) close to the chromosomal region identified in the present study (506-553Mb) (all chromosomal locations are based on IWGSC blast results). The marker RAC875_rep_c71463_98, located on 6B (699Mb; Zanke et al. 2014) also co-localized with the marker identified in the present study (709Mb), while the markers on 7B were located in the same region of the chromosome (Ex_c101666_634 (24.1Mb) in Zanke et al. (2014) vs. Kukri_c46310_84 $(0.1 \mathrm{Mb})$ in the present study). The sequence for the SSR marker on 1B GDM0126_196 (124.9 cM) identified by Zanke et al. (2014) was not available, rendering a full comparison of chromosomal locations impossible, but based on linkage maps of the SSR markers, GDM0126_196 seemed to be located in the middle of the chromosome upstream of the marker identified in the present study, which were located towards the telomere of chromosome 1B.. The observed difference in significant marker positions could be explained by differences in the germplasm between the studies. I the present study the majority of lines are semi-dwarfs (with respect to either Rht-B1b or Rht-D1b) compare to 7\% and 58\% respectively of the winter wheat varieties used in the study Zanke et al. (2014). Their germplasm also differed further as 14 spring varieties also were included in the analysis.

Lodging was scored between once and three times during the growth season, although only if relevant due to weather conditions (rain, hail and wind). Due to good weather conditions, there was no scoring of intermediate-stage lodging during 2015/2016. Significantly associated SNPs were identified for early lodging and late lodging, while for intermediate lodging only weak associations were found (Fig. 2 and Table 4). This indicates that the early and late stages of lodging were controlled by different genes, except for genes located on chromosome 5B at around 551.7712.9Mb. Intermediate stage lodging shared some tendencies with both the early and late stages and did not show any strong MTAs. It is therefore likely to be a less expressed trait, which might explain why it was harder to identify true MTAs for lodging at this stage. The absence of scores for intermediate-stage lodging in the final year (2015/16) also weakened the strength of the trait and may explain why it was difficult to identify true MTAs. 
The marker identified on chromosome 1B was identical between late lodging and plant height, and the markers found on 4B were at approximately the same locus 552.0-552.8Mb (Table 4). It is likely that the absence of the Rht-B1b allele in 4B had an impact on lodging because it is negatively affected by plant height (Verma et al. 2005). The marker identified on 1B for lodging was therefore likely to be a marker associated with plant height. Association between 1B and plant height has been demonstrated in other studies(Liu et al. 2011; Griffiths et al. 2012; Zanke et al. 2014). Verma et al. (2005) found associations between the width of cell walls in the first internode and chromosome 1B as well as a strong correlation between the length of the first internode and cell wall width. It is possible that the MTA observed in the present study was involved in the height and stem thickness of the first internode and therefore occurred as a significant marker for both plant height and lodging. The results indicate that plant height has a greater influence on lodging in the later developmental stages compared to the early growth stage (Table 4). Tendencies at intermediate stage lodging confirmed this theory since the same marker was identified as having weak associations in $1 \mathrm{~B}$ and a marker at approximately the same locus in $4 \mathrm{~B}$.

There have been few GWAS studies published on wheat lodging as a trait, but more studies have been conducted on association mapping/ QTL analysis. MTAs for lodging have been reported for chromosomes 3A and 3B when running GWAS with 1322 winter wheat lines and 15K SNP array (Edriss et al. 2017). In the present study, only 3B was identified as a MTA and only for early stage lodging. The association found on 1B, 4B, 6D, and 7D (Fig. 2) has previously been documented in at least two QTL studies on winter wheat (in one or both) (Verma et al. 2005; Zhang et al. 2008). A recent study that analyzed lodging-associated plant characteristics in winter wheat found markers on chromosomes 3A, 5B, and 6B that were associated with the root plate width, stem strength, stem wall width and stem diameter indicating that the loci found here might play a role in cell wall strength (Berry and Berry 2015). The MTAs on 5B identified for early and late stage lodging have not previously been associated with lodging, but based on the study by Berry and Berry (2015) they are likely to be involved in cell wall strength. Zhang et al. (2008) suggest that the MTA located on 7A represents an epistatic association for lodging between 7A and 4D, such that an effect in 7A is associated with the absence or presence of genes on 4D. However a direct link was not observed. To the authors' knowledge, MTAs for early lodging on chromosomes 5B, 6D and 7A and for late lodging on chromosomes 2A, 3D and 5B have not been reported and hence are likely to be novel markers. The strongest markers for lodging are those associated with chromosome 5B and these could be used in marker-assisted selection in breeding programmes to screen for lodging.

In conclusion, only the chromosome 5B markers were identical at all stages of lodging, indicating that this region could carry the same QTL due to the greater extent of linkage disequilibrium (LD) in modern wheat varieties (Hao et al. 2011; Giraldo et al. 2016). Moreover the three stages of lodging were likely to be controlled by different genes. Intermediate and late stage lodging seemed to be affected more by plant height, since they both mapped to the same SNP in 1B and to the same location in $4 \mathrm{~B}$ as identified for plant height.

Grain yield, harvest index, and STB

It was not possible to detect significant SNPs for grain yield , but the chromosomes that showed a weak association with grain yield (1A, 1B, 2A, 3A, 5B, 7A, 7B and 7D; Fig. 4) were in agreement with previous studies (Bordes et al. 2014; Edae et al. 2014; Assanga et al. 2017). Grain yield is a highly quantitative trait that is controlled by many minor 
and major genes and identification of new QTLs might be difficult in modern European wheat partly due to inbreeding and common genetic backgrounds.

The significantly associated MTAs for harvest index were not previously reported(Edae et al. 2014; Ain et al. 2015; Sukumaran et al. 2015) which could be due to differences in germplasm as all the above studies were based on panels of spring wheat varieties either in moist, temperated, stressed or rainfed climates. Furthermore, the panel in Ain et al. (2015) consisted of historical wheat cultivars from Pakistan, which are expected to have an even more diverse genetic background. No studies to our knowledge have been performed in winter wheat addressing MTAs for harvest index and the observed MTAs in chromosome 2A and 5B are therefore likely to be novel markers that can be tested in breeding programmes to improve the harvest index.

The markers identified for STB have already been documented in other studies (Brown et al. 2015; Arraiano and Brown 2017; Vagndorf et al. 2017), with some of them located either in the same or close to the loci of known Septoria tritici blotch (Stb) genes. The MTA in 3B was located close to the Stb14 gene (Cowling 2006), the MTA in 5B was located upstream of the Stb1 gene (Adhikari et al. 2004), and the MTA in 7A was at approximately the same locus as the Stb3 gene (Goodwin and Thompson 2011) and the TmStb1 gene (Brown et al. 2015).

Vagndorf et al. (2017) conducted GWAS with 15k SNP markers on a panel of 164 North European winter wheat varieties and breeding lines grown for three years at three locations in Denmark. They observed an association in 5D and 7A, with the association in 5D at approximately the same locus (560.5 Mb) as that observed in the present study. The association in 7A, however, was at opposite ends of the chromosome and the present finding on chromosome 7A was therefore more in agreement with the findings of Goodwin and Thompson (2011) and Brown et al. (2015).

The MTAs observed in 1A and 6B did not co-localize with any of the known Stb genes but did co-localized with the QTLs (QTL2 and MQTL21) identified by Brown (2015). The observed association in chromosome 5D was in the same region as a QTL observed in a later study (Arraiano and Brown 2017). Therefore, none of the identified MTAs for STB are novel markers, but they may still be important for STB-resistance in breeding.

Biorefinery traits: straw quality and yield

Difficulties in identifying MTAs are likely to be attributable to the low heritability of straw yield (Fig. 5). Only markers that were weakly associated $(\mathrm{p}<0.001)$ with straw yield were identified on chromosome $1 \mathrm{~B}$ and $4 \mathrm{~B}(552.8 \mathrm{Mb}$ and $1.3 \mathrm{Mb}$ respectively), indicating that these loci could play a role in controlling straw yield. The 1B loci were identical to those for plant height, corroborating the finding that plant height affects the yield of straw biomass. The association in 4B was not in the same chromosomal region as that for plant height (Table 4) but it might still be due to the presence or absence of the Rht-B1 gene. None of the weak associations have previously been documented (Sukumaran et al. 2015; Qaseem et al. 2018). Qaseem et al. 2018 conducted an association study on bread wheat, testing for a wide number of agronomic traits, including biomass, and the effects of drought and heat. For the control group, only one locus was identified and localized on chromosome 5A, while MTAs on chromosome 2B were identified under heat conditions, 3B under drought conditions and 2A, 5A, and 7A under combined heat and drought conditions. After conducting GWAS on a population of spring wheat, Sukumaran et al. (2015) showed an association between biomass and chromosome 3D and 6A at a significance level of 0.0001 . 
Both Sukumaran et al. (2015) and Qaseem et al. (2018) succeeded in identifying markers for biomass with a higher power $(\mathrm{p}<0.0001)$ than that documented in the present study $(\mathrm{p}<0.001)$. One explanation is obviously a difference in mapping populations (spring wheat vs. winter wheat) but in addition the study conducted by Qaseem et al. (2018) was a pot experiment performed in a greenhouse with a smaller influence of variation in environmental factors compared to a field experiment. However, a greenhouse environment might not reflect the environment in the field, and the MTAs identified might therefore differ between the two. A recent study also conducted on spring wheat in Kazakhstan also failed to identify any MTAs for biomass using a diverse panel of 194 lines at three locations over three years (Turuspekov et al. 2017). The number of lines was similar to that used in the present study, which could indicate that the lack of identified MTAs is due to the number of lines being too small. Biomass yield is also affected by other traits such as plant height, and controlling for this, by including plant height as a covariate in the mixed linear model in GWAS, might make it easier to detect significant MTAs. Moreover, like grain yield, straw yield is a quantitative trait possibly controlled by several small effector genes, and this may also have contributed to the difficulties in identifying significant MTAs for straw yield.

Despite the low heritability observed for straw quality traits (glucose, xylose and arabinose release), MTAs for all three monosaccharides were identified, which all had a pronounced effect when converted to kg monosaccharide per hectare. None of the observed MTAs for monosaccharide release were identical between the monosaccharide types. However, the markers in chromosome 6A were located in approximately the same chromosomal region (597.9, 429.4, and $524.8 \mathrm{Mb}$ for glucose, xylose and arabinose respectively). It is therefore likely that there are genes in this region that affect the cell wall structure, making the hemicellulose and cellulose more accessible for enzymes, such that sugar release is increased. The fact that all monosaccharide release yield were positively correlated also supports this finding (Table 5). Of the 108 cellulose synthase (Csl) genes identified in wheat, three are located on 6A which could directly control the structure of hemicellulose (Kaur et al. 2017). Alternatively, it could be the genes involved in cell wall composition (causing changes in lignin, hemicellulose structure or compactness) or those directly involved in cell wall bindings, such that the cell wall becomes more open and easily accessible. Further studies are needed to clarify the impact of chromosome 6A on releasing monosaccharides from straw.

The markers identified in 1B for glucose and in 4B for arabinose were not identical with those found for plant height, but the MTA identified in 1B for glucose might be located in the same chromosomal region due to large LD observed in wheat (Giraldo et al. 2016), i.e. 619.7Mb and 557.4Mb for glucose and plant height. The markers for arabinose and plant height (377.8Mb and 506.1 Mb respectively) were located further apart and are not likely to be associated with the same genes. These findings indicate that plant height might also affect the yield of released glucose (Table 4).

To the best of the authors' knowledge, only one association study on sugar release after enzymatic saccharification has been conducted to date (Bellucci et al. 2015). In that study, only weak MTAs for glucose release were identified on chromosome 1B. One of the MTAs for glucose release documented in the present study was also localized on chromosome 1B and in approximately the same location (Bellucci et al. 2015). These two studies used different saccharification methods on completely different wheat germplasm, which strongly supports the genes on chromosome $1 \mathrm{~B}$ being involved in regulating glucose release. 
In the present study, four MTAs were observed for xylose release and four for arabinose release, while none were identified in a study by Bellucci et al. (2015). It is likely that these chromosomes have a role in controlling the hemicellulose structure (arabinose and xylose modifications), such that they can be accessed more easily by enzymes, which in turn will contribute to increasing sugar release. More studies are needed to verify this. Both xylose and arabinose are part of the hemicellulose structure in the cell wall, and this complex structure is presumably controlled by many small effector genes, which also explains the small effect size (See Figure 6 and Table 4). In addition, the experimental protocol used for the sugar release assay may be highly important in identifying MTAs for sugar release (Oakey et al. 2013; Lindedam et al. 2014; Jørgensen et al. 2018) and further experimental optimization may increase the possibility of identifying them. Especially the noise introduced during the pretreatment, the enzymatic hydrolysis and/or the quantification of monosaccharides should be reduced, to obtain higher heritability estimates and to increase the chance of identifying MTAs with bigger effect sizes. Although the effect size seemed low, it still would correspond to and pronounced increase in sugar release in kilograms per hectare and especially if the effect were additive (Table 6).

\section{Controlling for plant height in GWAS}

Many of the traits analyzed by GWAS, including lodging, straw yield and glucose seemed to be affected by plant height. To verify whether plant height was indeed the factor affecting these traits, GWAS was conducted with plant height included as a covariate.

Some of the MTAs identified for late lodging seemed to be affected by plant height, while early-stage lodging was unaffected (MTAs in 1B and 4B; Table 4). This difference in plant height dependency between late and early lodging was partly confirmed by controlling for plant height in GWAS, since MTAs for early lodging were almost unaffected while the MTAs and weak MTAs for late and intermediate lodging, respectively, were greatly affected (Table 7). For early lodging it was only the significance level that changed, where the $-\log 10(p)$ was higher for 3B and 5B and lower for 6A,7D and 7A. The MTA in 7A was actually below the significance tradeoff level corresponding to a -log10(p) of 5.05 (Table 7). Early lodging was therefore not dependent on plant height and its inclusion as a covariate did not improve the QQ-plot (results not shown).

The weak associations observed for intermediate lodging were greatly affected by plant height, since chromosomes 1B, 2B and 3B were now the only chromosomes with weak associations, eliminating weak associations in 4B, 5B, and 7A. $1 \mathrm{~B}$ was still weakly associated, and even thought the markers differed, two of them mapped to the same chromosomal region (502.5Mb and 548.7Mb for wsnp_Ra_c5088_9081927 and Tdurum_contig42749_3287, respectively) while the last marker BS00064032_51 was located at the start of the chromosome (144.9Mb) (Tables 4 and 5). Based on this, the association in 1B might not only be an effect of plant height.

The MTAs for late lodging also differed, with associations found in 6B and 5D (705.0Mb and 341.6Mb, respectively), while associations in 1B and 4B became weak associations. None of the MTAs identified for late lodging were identical with the MTAs identified without plant height as a covariate, however the weak associations identified in $1 \mathrm{~B}$ (520.5Mb) and $4 \mathrm{~B}(609.3 \mathrm{Mb})$ were in the same chromosome region. Therefore plant height seemed not to be the only factor responsible for the associations in $1 \mathrm{~B}$ and $4 \mathrm{~B}$. Moreover, the introduction of plant height as a covariate did not improve the QQ-plot for intermediate and late lodging, and therefore the results in Table 4 are the most reliable. Association in 1B and 4B for late lodging are therefore likely to be novel markers for lodging and not just an effect of plant height. 
685 When plant height was included as a covariate for glucose release, the effect of the MTAs in 1B disappeared, however 686 the other effects all disappeared as well (Table 7). The QQ-plots indicated that the GWAS model that best described 687 data was the one in which plant height was not included as a covariate, which is why the correct MTA results are those 688 given in Table 4.

689 In conclusion, controlling for plant height did not improve the QQ-plots for any of the affected traits and plant height

690 did not seem to be the major QTLs identified in 1B and 4B. The markers are therefore likely to be novel markers for the 691 traits investigated. 
Table 5: Comparison of MTAs identified by GWAS with and without plant height as covariate

\begin{tabular}{|c|c|c|c|c|c|c|c|}
\hline \multirow[t]{2}{*}{ Trait } & \multicolumn{3}{|l|}{ Standard GWAS } & \multicolumn{4}{|c|}{ GWAS with plant height as covariate } \\
\hline & Marker type & Chr. $^{\mathrm{a}}$ & $-\log 10(p)$ & Marker type & Chr. $^{\mathrm{a}}$ & Pos. $[\mathrm{Mb}]^{\mathrm{a}}$ & $-\log 10(p)$ \\
\hline Early & wsnp_Ex_c9362_15546626 & $5 B$ & 19.19 & wsnp_Ex_c9362_15546626 & $5 B$ & 693.6 & 20.57 \\
\hline \multirow[t]{5}{*}{ lodging } & BS00009777_51 & $5 B$ & 18.34 & BS00009777_51 & $5 B$ & 712.9 & 19.01 \\
\hline & GENE-3659_162 & $6 \mathrm{~A}$ & 9.56 & GENE-3659_162 & $6 \mathrm{~A}$ & 101.3 & 8.84 \\
\hline & Kukri_c5789_1029 & 7D & 8.55 & wsnp_Ex_c123_244117 & $3 B$ & 485.4 & 8.12 \\
\hline & wsnp_Ex_c123_244117 & 3B & 7.18 & Kukri_c5789_1029 & $7 \mathrm{D}$ & 605.0 & 7.09 \\
\hline & Ra_c31466_694 & 7A & 6.57 & Ra_c31466_694 & $7 \mathrm{~A}$ & 586.2 & 4.83 \\
\hline \multirow{5}{*}{$\begin{array}{l}\text { Intermediate } \\
\text { lodgingb }^{\text {bdying }}\end{array}$} & CAP8_c3273_136 & 4B & 3.41 & IACX1098 & $2 \mathrm{~B}$ & 58.3 & 3.36 \\
\hline & BS00075731_51 & $7 \mathrm{~A}$ & 3.13 & RFL_Contig1456_842 & $3 \mathrm{~B}$ & 39.9 & 3.36 \\
\hline & Jagger_c505_141 & $5 B$ & 3.11 & wsnp_Ra_c5088_9081927 & $1 \mathrm{~B}$ & 502.5 & 3.36 \\
\hline & Ku_c18920_414 & $1 \mathrm{~B}$ & 3.02 & Tdurum_contig42749_3287 & $1 \mathrm{~B}$ & 548.7 & 3.36 \\
\hline & & & & BS00064032_51 & $1 \mathrm{~B}$ & 144.9 & 3.36 \\
\hline \multirow[t]{6}{*}{ Late lodging } & Tdurum_contig91519_224 & $2 \mathrm{~A}$ & 8.56 & BS00046264_51 & $6 \mathrm{~B}$ & 705.0 & 5.76 \\
\hline & Ku_c18920_414 & $1 \mathrm{~B}$ & 6.86 & GENE-3583_319 & $5 \mathrm{D}$ & 341.6 & 5.49 \\
\hline & CAPי8_c3273_136 & $4 \mathrm{~B}$ & 6.61 & wsnp_Ex_c7988_13555376 & $5 B$ & 316.7 & 4.44 \\
\hline & BS00082101_51 & $5 B$ & 5.63 & BS00057153_51 & $4 \mathrm{~B}$ & 609.3 & 4.03 \\
\hline & GENE-2712_130 & $3 \mathrm{D}$ & 5.12 & BS00091666_51 & $6 \mathrm{~A}$ & 31.4 & 3.55 \\
\hline & RAC875_rep_c105937_467 & $6 \mathrm{D}$ & 5.11 & wsnp_Ra_c5088_9081927 & $1 \mathrm{~B}$ & 520.5 & 3.47 \\
\hline \multirow[t]{2}{*}{ Glucose } & wsnp_Ex_c18384_27213023 & $1 \mathrm{~B}$ & 7.07 & & & & \\
\hline & wsnp_Ex_c2236_4189774 & $6 \mathrm{~A}$ & 5.84 & & & & \\
\hline
\end{tabular}

$693 \quad{ }^{a}$ The chromosomal position of the SNP is obtained from BLAST of the marker sequence applying the new IWGSC RefSeq v1.0 sequence available at

694 https://urgi.versailles.inra.fr/blast_iwgsc/?dbgroup=wheat_iwgsc_refseq_v1_chromosomes\&program=blastn and marker sequences at

695 https://triticeaetoolbox.org/wheat/. For exact chromosomal locations for standard GWAS results please refer to Table 4.

696 Markers in grey are identical for the two different GWAS methods.

$697 \quad{ }^{\mathrm{b}}$ MTAs are based on week associations and are not true MTAs 
Applying GWAS on lines from an active breeding nursery Using an active breeding nursery for GWAS was highly effective at identifying MTAs, but due to limited variation in more inbred traits, the genetic effect was expected to be relatively small. This was clearly also the case for grain yield where no markers were detected. The lines included in the breeding programme had been bred primarily for yield, so the variation should have been relatively low. However, the obtained grain yield still exhibited a relatively high genetic variance (9.48) and heritability (0.37). In theory, this should increase the likelihood of detecting MTAs, but since grain yield is controlled by many small QTLs, it may be difficult to detect the associated chromosomes even though heritability is present.

Using rotation in breeding nurseries that replace $50 \%$ of the lines each year is both an advantage and a disadvantage. The advantage is that it increases the number of lines included in GWAS, hence increasing its power, but the disadvantage is it increases the $\mathrm{G} \times \mathrm{E}$ variance, making identification of the same markers in the three consecutive years difficult. Despite the use of rotation and the lack of MTAs for grain yield, straw yield, and intermediate lodging MTAs for all the other traits analysed, including plant height, were still obtained. It may now be common practice in breeding companies to conduct GWAS in an active breeding nursery rather than using a designed population but this is not widely published and here it shows indeed to be highly versatile at identifying MTAs through GWAS.

Using active breeding nurseries for the identification of dual-purpose varieties with a high yield of both grain and straw is a challenge, as demonstrated by Townsend et al. (2017) and as confirmed in the present study with the difficulties in identifying MTAs for straw and grain yield. However, it was still possible to identify MTAs for biorefinery traits in the lines bred for a high grain yield, indicating that dual-purpose varieties may be indeed obtained without the introduction of more distinct germplasm. Landraces that can add greater biomass variation may be desirable for breeding dualpurpose varieties, but this will also introduce undesirable MTAs related to height, which will need to be removed in order to develop competitive cultivars, and therefore it probably will have limited use in later stages of breeding.

Challenges in identifying genetic effects

The detection of a genetic effect was challenging for the majority of the traits despite the fact that MTAs were identified in most cases (Tables 3 and 4). Heritability obtained by mixed linear models was considered low for early stage lodging, harvest index and release of the three monosaccharides after enzymatic hydrolysis $\left(H^{2}<0.15\right)($ Table 3$)$. Repeatability, which were based on the genetic variance, the genotype by year variance and the error term (like heritability) were divided with the number of repeats and number of years and were therefore considerably higher than heritability (Table 3).

Repeatability was much higher than heritability for straw yield for which no MTAs were identified $\left(H^{2}\right.$ of 0.15 , Table 3). Other studies have experienced similar difficulties in obtaining high heritability and repeatability for straw yield (Bellucci et al. 2015; Sukumaran et al. 2015). Regardless of the low heritability, Sukumaran et al. (2015) still succeeded in identifying MTAs for biomass yield for spring wheat in the controlled glasshouse environment andonspring wheat. No MTAs for grain yield and straw yield were identified in the present study, which may be explained by the fact that both traits are considered to be highly quantitative and that little variation existed in the analysed germplasm. However, both the heritability and the repeatability for grain yield were high indicating that there indeed was genetic variation in the germplasm even though it was not possible to identify MTAs. The number of varieties used in the present study 
might be too low to capture the variation because grain yield is known to be controlled by many small and major effector genes. The $\mathrm{G} \times \mathrm{E}$ variance was also high for grain yield as it explained about $43 \%$ of the total variation (based on genetic, $\mathrm{G} \times \mathrm{E}$ and error variance components) indicating that gain yield also were greatly affected by the environment or the rotation of varieties each year. Furthermore, yield and grain yield gave a perfect fit which calls for developing other algorithms or including other co-variates that capture the population structure better to increase the chance of identifying MTAs for these traits.

Heritability was relatively low for early-stage lodging and increased at each developmental stage (Table 3), nevertheless MTAs were only identified for the early and late developmental stages (Table 4). All three stages of lodging were greatly affected by the environment as lodging is mainly initiated by rainy and windy weather conditions (Pinthus 1974). Plants that were lodging at an early stage might be standing in the final growth stage because plants have the ability to rise again. This is also apparent in the relatively high $\mathrm{G} \times \mathrm{E}$ components for lodging (Table 3) which are explaining between 23 and $46 \%$ of the total variation with the $\mathrm{G} \times \mathrm{E}$ variance increasing with the stage of lodging (early, intermediate and late). The severity of lodging at a late development stage was expected to be more dependent on plant genetics since changes in the plants physical architecture would be more prominent. The combination of plant height, root development, stem strength and seed weight was therefore likely to have a greater effect at the intermediate or late development stages compared to the early stages (Verma et al. 2005; Berry and Berry 2015). This was also confirmed by the association between the observed MTAs for late and intermediate-stage (weak associations) lodging and plant height (Table 4). MTAs for intermediate and late-stage lodging can therefore be harder to detect, even when high heritability is present, because they seem to be more complex traits that are considerably affected by interactions between weather conditions and genetics.

For almost every trait (with the exception of plant height, STB and xylose), the $\mathrm{G} \times \mathrm{E}$ effect was greater than the genetic effect. It was therefore harder to find associated SNPs that were fully consistent between years. The fact that 50 lines were excluded and 50 new ones were introduced each year may be a reasonable explanation for increasing the $\mathrm{G} \times \mathrm{E}$ variance. Using an active breeding nursery with rotation for MTA analysis therefore makes it difficult to find stable markers throughout years.

The low heritability for sugar release was primarily due to variance caused by analytical errors, as the error variance explained the majority of the variance in the model (around $90 \%$ of the total variance). The identification of genetic variance in sugar release between cultivars was therefore highly challenged by the analytical variance introduced. It was not clear which step in the chemical/enzymatic treatment was responsible for the analytical variation. Even though, variation from plate to plate and between enzyme batches might be the main contributors. To normalize data for plateto-plate variation, control samples were included in every plate, but this was not sufficient to account completely for the experimental variance. Another study has shown that the organization of the experiment and the location of samples on the plate was important for detecting heritability (Oakey et al. 2013). By including these parameters in the mixed linear model applied, Oakey et al. (2013) succeeded in correcting for this variance and increase the heritability. In the present study, plate position and plate number were included as explanatory random variables in the mixed linear model to adjust for this variance. Despite the adjustment, heritability was still low. The plate position did have an impact, however the effect was small compared to the variance introduced by the plate number (results not shown). In future 
studies, reducing the plate-to-plate variation might be one way of increasing the heritability for release of monosaccharides after enzymatic saccharification. Despite the difficulties in obtaining high heritability for the release of monosaccharides, it was still possible to obtain MTAs, where for glucose one of the MTAs was identical with a locus previously identified by Bellucci et al. (2015).

Therefor heritability and repeatability are not always correlated with the ability to detect MTAs. Instead, heritability gives information about how much of the phenotypic variation is due to genetic variation and how much of any variation can be theoretically explained by an SNP effect.

\section{Conclusions}

This study demonstrates that the GWAS algorithm FarmCPU can be applied successfully in an active breeding nursery, thereby providing identification of MTAs for a number of agronomic traits including plant height, lodging, STB susceptibility, and harvest index. Significant MTAs for glucose, xylose, and arabinose release from straw by enzymatic saccharification were identified, demonstrating that it is possible to breed for biorefinery-related traits.

\section{References}

Adhikari TB, Yang X, Cavaletto JR, et al (2004) Molecular mapping of Stb1, a potentially durable gene for resistance to septoria tritici blotch in wheat. Theor Appl Genet 109:944-953. doi: 10.1007/s00122-004-1709-6

Ain Q-U, Rasheed A, Anwar A, et al (2015) Genome-wide association for grain yield under rainfed conditions in historical wheat cultivars from Pakistan. Front Plant Sci 6:743. doi: 10.3389/fpls.2015.00743

Akhunov E, Nicolet C, Dvorak J (2009) Single nucleotide polymorphism genotyping in polyploid wheat with the Illumina GoldenGate assay. Theor Appl Genet 119:507-517. doi: 10.1007/s00122-009-1059-5

Arraiano LS, Brown JKM (2017) Sources of resistance and susceptibility to Septoria tritici blotch of wheat. Mol Plant Pathol 18:276-292. doi: 10.1111/mpp.12482

Assanga SO, Fuentealba M, Zhang G, et al (2017) Mapping of quantitative trait loci for grain yield and its components in a US popular winter wheat TAM 111 using 90K SNPs. PLoS One 12:e0189669. doi: 10.1371/journal.pone.0189669

Bellucci A, Torp AM, Bruun S, et al (2015) Association Mapping in Scandinavian Winter Wheat for Yield, Plant Height, and Traits Important for Second-Generation Bioethanol Production. Front Plant Sci 6:1-12. doi: 10.3389/fpls.2015.01046

Berry PM, Berry • S T (2015) Understanding the genetic control of lodging-associated plant characters in winter wheat (Triticum aestivum L.). Euphytica - Springer 671-689. doi: 10.1007/s10681-015-1387-2

Bordes J, Goudemand E, Duchalais L, et al (2014) Genome-wide association mapping of three important traits using bread wheat elite breeding populations. Mol Breed 33:755-768. doi: 10.1007/s11032-013-0004-0 
Börner A, Plaschke J, Korzun V, Worland AJ (1996) The relationships between the dwarfing genes of wheat and rye. Euphytica 89:69-75. doi: 10.1007/BF00015721

Brading PA, Verstappen CP, Kema GHJ, Brown JKM (2002) Genetics and Resistance A Gene-for-Gene Relationship Between Wheat and Mycosphaerella graminicola, the Septoria Tritici Blotch Pathogen. Phytopathology 92:439. doi: 10.1094/PHYTO.2002.92.4.439

Brown JKM, Chartrain L, Lasserre-Zuber P, Saintenac C (2015) Genetics of resistance to Zymoseptoria tritici and applications to wheat breeding. Fungal Genet Biol 79:33-41. doi: 10.1016/j.fgb.2015.04.017

Cowling SG (2006) Identification and mapping of host resistance genes to Septoria tritici blotch of wheat. The University of Manitoba

Crossa J, Burgueño J, Dreisigacker S, et al (2007) Association Analysis of Historical Bread Wheat Germplasm Using Additive Genetic Covariance of Relatives and Population Structure. Genetics 177:1889-1913. doi: 10.1534/genetics.107.078659

Dalgaard T, Hansen B, Hasler B, et al (2014) Policies for agricultural nitrogen management-trends, challenges and prospects for improved efficiency in Denmark Related content Synthesis and review: Tackling the nitrogen management challenge: from global to local scales. Environ Res Lett 9:. doi: 10.1088/1748-9326/9/11/115002

Edae EA, Byrne PF, Haley SD, et al (2014) Genome-wide association mapping of yield and yield components of spring wheat under contrasting moisture regimes. Theor Appl Genet 127:791-807. doi: DOI 10.1007/s00122-013-22578

Edriss V, Cericola F, Orabi J, et al (2017) Comparing single trait with multi-trait genome-wide association in winter wheat (Triticum aestivum). In: Buerstmayr H, Lang-Mladek C, Steiner B, et al. (eds) Proceedings 13th International Wheat Genetics Symposium. BOKU - University of Natural Resources and Life Sciences, Vienna Department of Agrobiotechnology (IFA-Tulln) \& Department of Crop Sciences, Tulln Austria, p 419

Gent MPN, Kiyomoto RK (1997) Physiological and Agronomic Consequences of Rht Genes in Wheat. J Crop Prod 1:27-46. doi: 10.1300/

Giraldo P, Royo C, González M, et al (2016) Genetic Diversity and Association Mapping for Agromorphological and Grain Quality Traits of a Structured Collection of Durum Wheat Landraces Including subsp. durum, turgidum and diccocon. PLoS One 11:e0166577. doi: 10.1371/journal.pone.0166577

Goodwin SB, Thompson I (2011) Development of Isogenic Lines for Resistance to Septoria Tritici Blotch in Wheat. Czech J Genet Plant Breed 47:S98-S101. doi: DOI: 10.17221/3262-CJGPB

Griffiths S, Simmonds J, Leverington M, et al (2012) Meta-QTL analysis of the genetic control of crop height in elite European winter wheat germplasm. Mol Breed 29:159-171. doi: 10.1007/s11032-010-9534-x

Hao C, Wang L, Ge H, et al (2011) Genetic Diversity and Linkage Disequilibrium in Chinese Bread Wheat (Triticum 
Hedden P (2003) The genes of the Green Revolution. Trends Genet 19:5-9. doi: 10.1016/S0168-9525(02)00009-4

Jørgensen H, van Hecke J, Zhang H, et al (2018) Wheat as a dual crop for biorefining: Straw quality parameters and their interactions with nitrogen supply in modern elite cultivars. GCB Bioenergy 11:400-411. doi: 10.1111/gcbb. 12560

Kaur S, Dhugga KS, Beech R, Singh J (2017) Genome-wide analysis of the cellulose synthase-like (Csl) gene family in bread wheat (Triticum aestivum L.). BMC Plant Biol 17:193. doi: 10.1186/s12870-017-1142-z

Korzun V, Röder MS, Ganal MW, et al (1998) Genetic analysis of the dwarfing gene (Rht8) in wheat. Part I. Molecular mapping of Rht8 on the short arm of chromosome 2D of bread wheat (Triticum aestivum L.). Theor Appl Genet 96:1104-1109. doi: 10.1007/s001220050845

Leplat F, Jensen J, Madsen P (2016) Genomic Prediction of Manganese Efficiency in Winter Barley. Plant Genome 9:0. doi: 10.3835/plantgenome2015.09.0085

Lindedam J, Bruun S, Jørgensen H, et al (2014) Evaluation of high throughput screening methods in picking up differences between cultivars of lignocellulosic biomass for ethanol production. Biomass and Bioenergy 66:261267. doi: 10.1016/j.biombioe.2014.03.006

Lipka AE, Tian F, Wang Q, et al (2012) GAPIT: Genome association and prediction integrated tool. Bioinformatics 28:2397-2399. doi: 10.1093/bioinformatics/bts444

Liu G, Xu SB, Ni Z, et al (2011) Molecular dissection of plant height QTLs using recombinant inbred lines from hybrids between common wheat (Triticum aestivum L.) and spelt wheat (Triticum spelta L.). Chinese Sci Bull 56:1897-1903. doi: 10.1007/s11434-011-4506-z

Liu X, Huang M, Fan B, et al (2016) Iterative Usage of Fixed and Random Effect Models for Powerful and Efficient Genome-Wide Association Studies. PLOS Genet 12:1-24. doi: 10.1371/journal.pgen.1005767

Oakey H, Shafiei R, Comadran J, et al (2013) Identification of crop cultivars with consistently high lignocellulosic sugar release requires the use of appropriate statistical design and modelling. Biotechnol Biofuels 6:185. doi: 10.1186/1754-6834-6-185

Ogbonnaya FC, Rasheed A, Okechukwu EC, et al (2017) Genome-wide association study for agronomic and physiological traits in spring wheat evaluated in a range of heat prone environments. Theor Appl Genet 130:1819-1835. doi: 10.1007/s00122-017-2927-z

Pearce S, Saville R, Vaughan SP, et al (2011) Molecular characterization of Rht-1 dwarfing genes in hexaploid wheat. Plant Physiol 157:1820-1831. doi: 10.1104/pp.111.183657

Pinthus MJ (1974) Lodging in Wheat, Barley, and Oats: The Phenomenon, its Causes, and Preventive Measures. Adv 
Qaseem MF, Qureshi R, Muqaddasi QH, et al (2018) Genome-wide association mapping in bread wheat subjected to independent and combined high temperature and drought stress. PLoS One 13:1-22. doi: 10.1371/journal

Selig MJ, Tucker MP, Sykes RW, et al (2010) Lignocellulose recalcitrance screening by integrated high-throughput hydrothermal pretreatment and enzymatic saccharification. Ind Biotechnol 6:104-111. doi: 10.1089/ind.2010.0009

Studer MH, DeMartini JD, Brethauer S, et al (2010) Engineering of a high-throughput screening system to identify cellulosic biomass, pretreatments, and enzyme formulations that enhance sugar release. Biotechnol Bioeng 105:231-238. doi: 10.1002/bit.22527

Sukumaran S, Dreisigacker S, Lopes M, et al (2015) Genome-wide association study for grain yield and related traits in an elite spring wheat population grown in temperate irrigated environments. Theor Appl Genet 128:353-363. doi: 10.1007/s00122-014-2435-3

Thomas SG (2017) Novel Rht-1 dwarfing genes: tools for wheat breeding and dissecting the function of DELLA proteins. J Exp Bot 68:354-358. doi: 10.1093/jxb/erw509

Townsend TJ, Roy J, Wilson P, et al (2017) Food and bioenergy: Exploring ideotype traits of a dual-purpose wheat cultivar. F Crop Res 201:210-221. doi: 10.1016/J.FCR.2016.11.007

Troyanskaya O, Cantor M, Sherlock G, et al (2001) Missing value estimation methods for DNA microarrays. Bioinformatics 17:520-525. doi: 10.1093/bioinformatics/17.6.520

Turuspekov Y, Baibulatova A, Yermekbayev K, et al (2017) GWAS for plant growth stages and yield components in spring wheat (Triticum aestivum L.) harvested in three regions of Kazakhstan. BMC Plant Biol 17:52-61. doi: 10.1186/s12870-017-1131-2

Vagndorf N, Nielsen NH, Edriss V, et al (2017) Genomewide association study reveals novel quantitative trait loci associated with resistance towards Septoria tritici blotch in North European winter wheat. Plant Breed 136:474482. doi: 10.1111/pbr.12490

van Grinsven HJM, ten Berge HFM, Dalgaard T, et al (2012) Management, regulation and environmental impacts of nitrogen fertilization in northwestern Europe under the Nitrates Directive; a benchmark study. Biogeosciences 9:5143-5160. doi: 10.5194/bg-9-5143-2012

Verma V, Worland AJ, Sayers EJ, et al (2005) Identification and characterization of quantitative trait loci related to lodging resistance and associated traits in bread wheat. Plant Breed 124:234-241. doi: 10.1111/j.14390523.2005.01070.x

Visscher PM, Hill WG, Wray NR (2008) Heritability in the genomics era - concepts and misconceptions. Nat Rev Genet 9:255-266. doi: 10.1038/nrg2322 
Wang Q, Tian F, Pan Y, et al (2014) A SUPER powerful method for genome wide association study. PLoS One 9:e107684. doi: 10.1371/journal.pone.0107684

Würschum T, Langer SM, Longin CFH (2015) Genetic control of plant height in European winter wheat cultivars. Theor Appl Genet 128:865-874. doi: 10.1007/s00122-015-2476-2

Zanke CD, Ling J, Rg Plieske J, et al (2014) Whole Genome Association Mapping of Plant Height in Winter Wheat (Triticum aestivum L.). PLoS One 9:e113287. doi: 10.1371/journal.pone.0113287

Zhang H, Fangel JU, Willats WGT, et al (2014) Assessment of leaf/stem ratio in wheat straw feedstock and impact on enzymatic conversion. GCB Bioenergy 6:90-96. doi: 10.1111/gcbb.12060

Zhang K-P, Zhao L, Hai Y, et al (2008) QTL Mapping for Adult-Plant Resistance to Powdery Mildew, Lodging Resistance, and Internode Length Below Spike in Wheat. Acta Agron Sin 34:1350-1357. doi: 10.1016/S18752780(08)60046-2

Zhang L, Xu X, Zhao C, et al (2011) QTL Analysis of Plant Height based on Doubled Haploid (DH) Population derived from PTSMS Wheat. Mol Plant Breed 2:92-97. doi: 10.5376/mpb.2011.02.0013

Zhang Z, Ersoz E, Lai C-Q, et al (2010) Mixed linear model approach adapted for genome-wide association studies. Nat Genet 42:355-60. doi: 10.1038/ng.546 
a
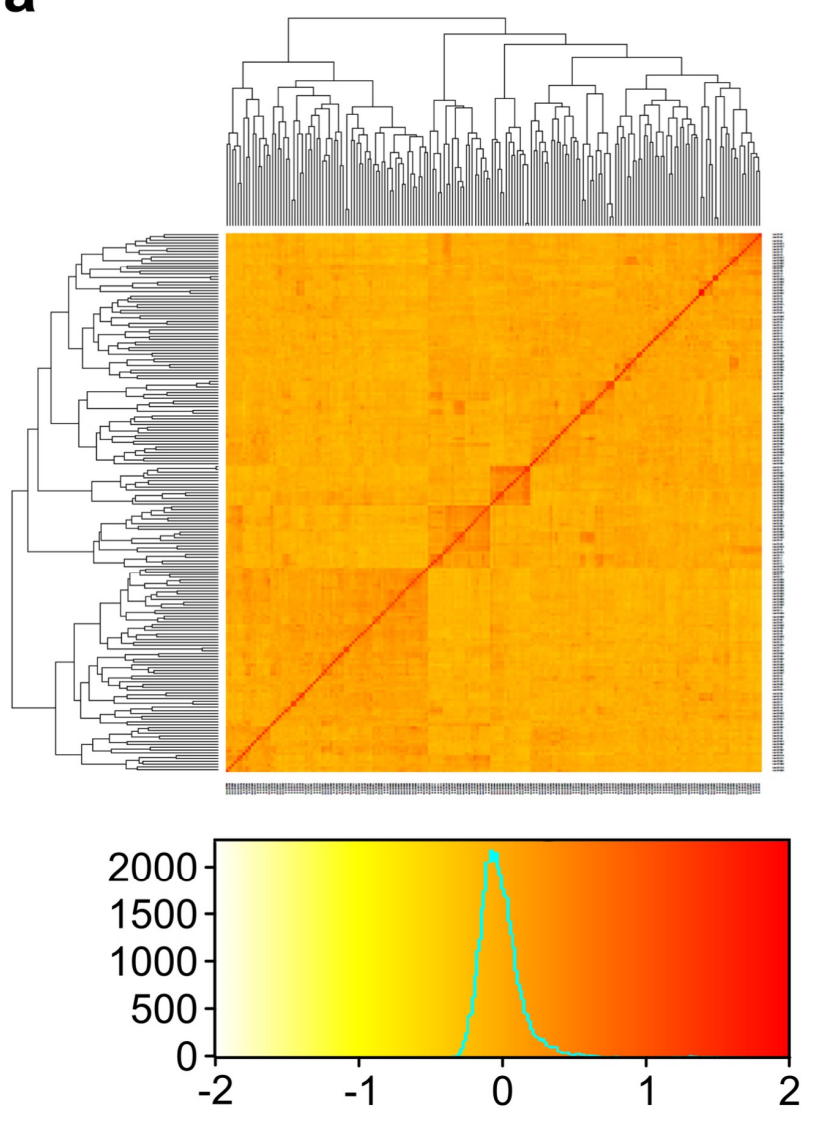

d

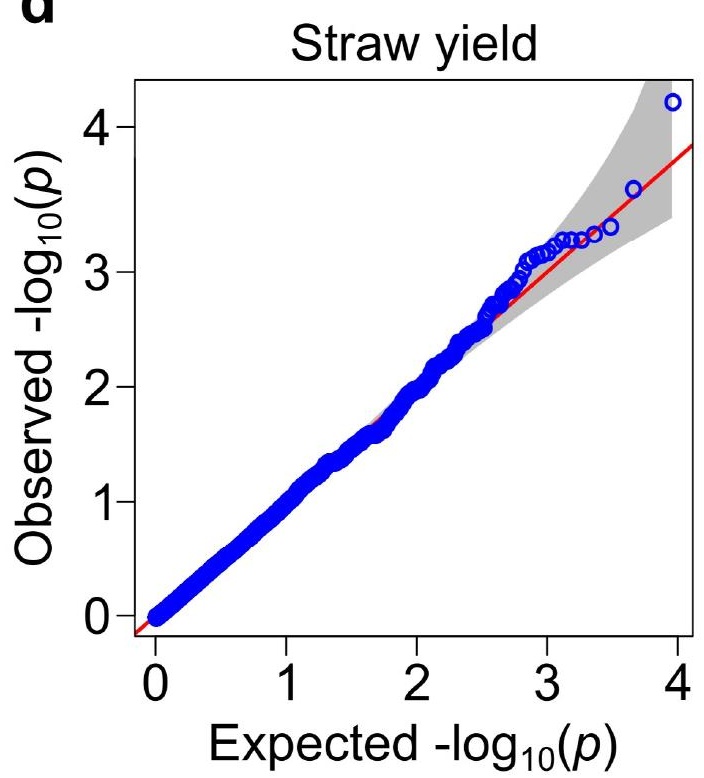

b
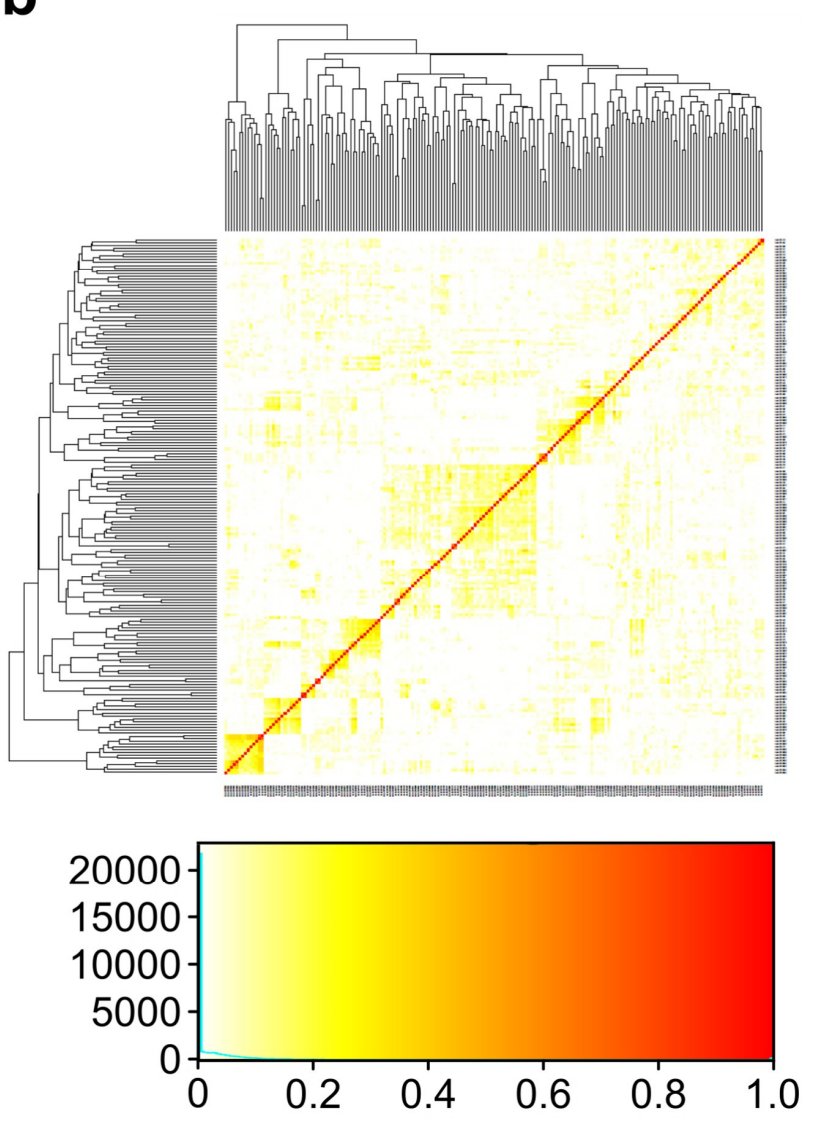

e

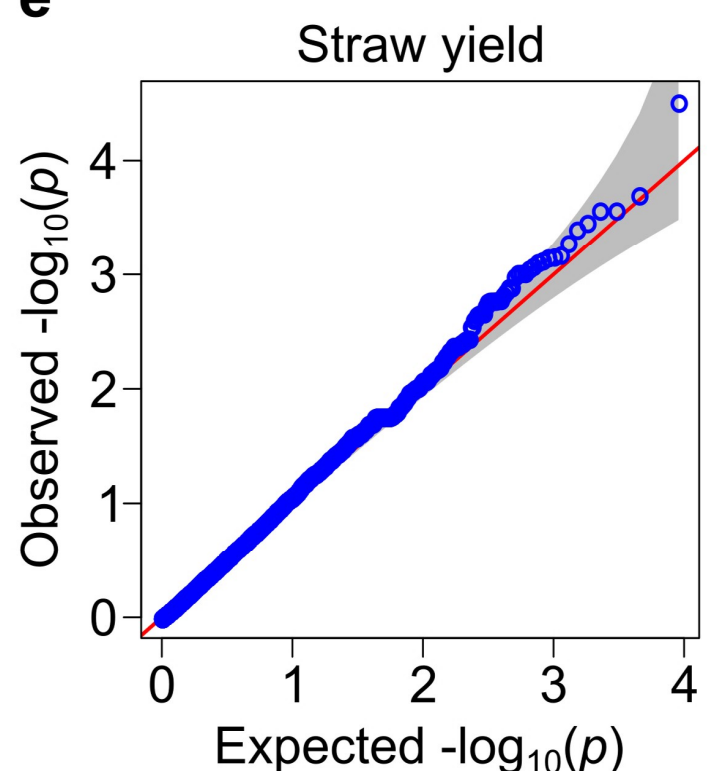

C
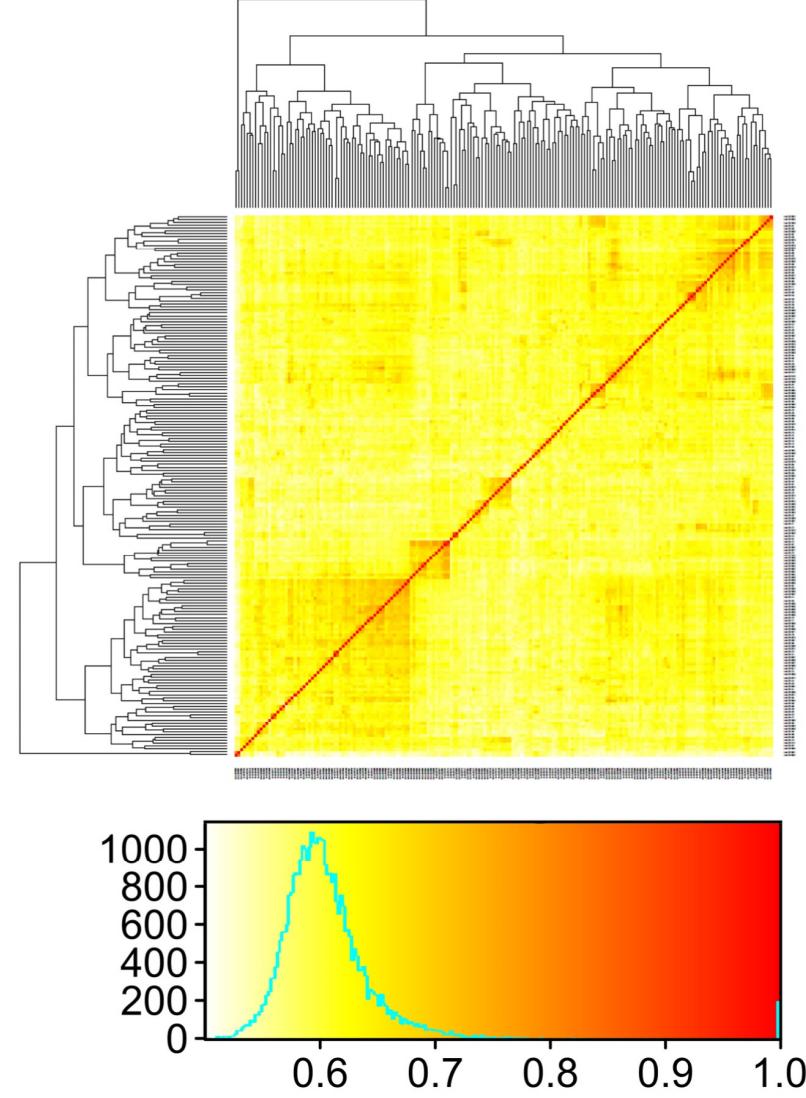

f
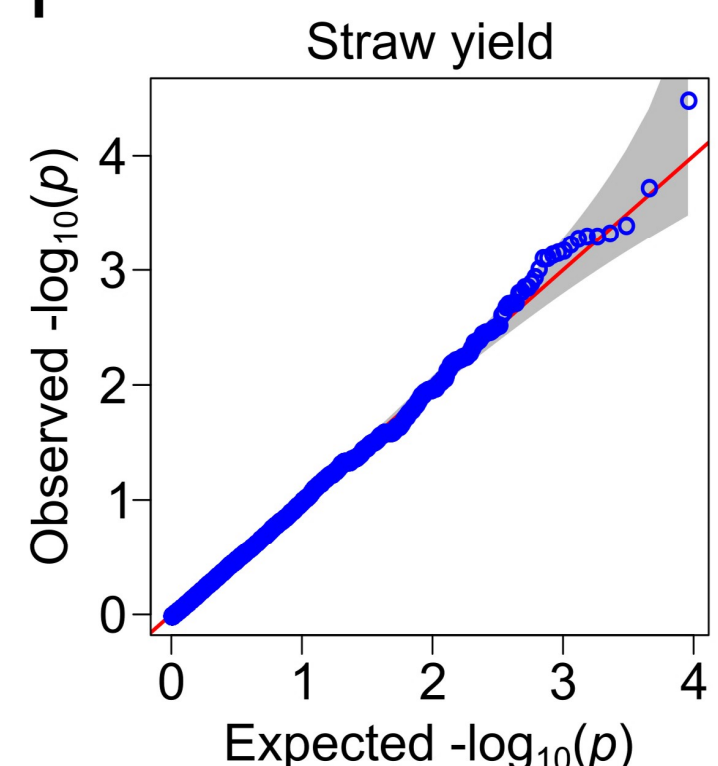


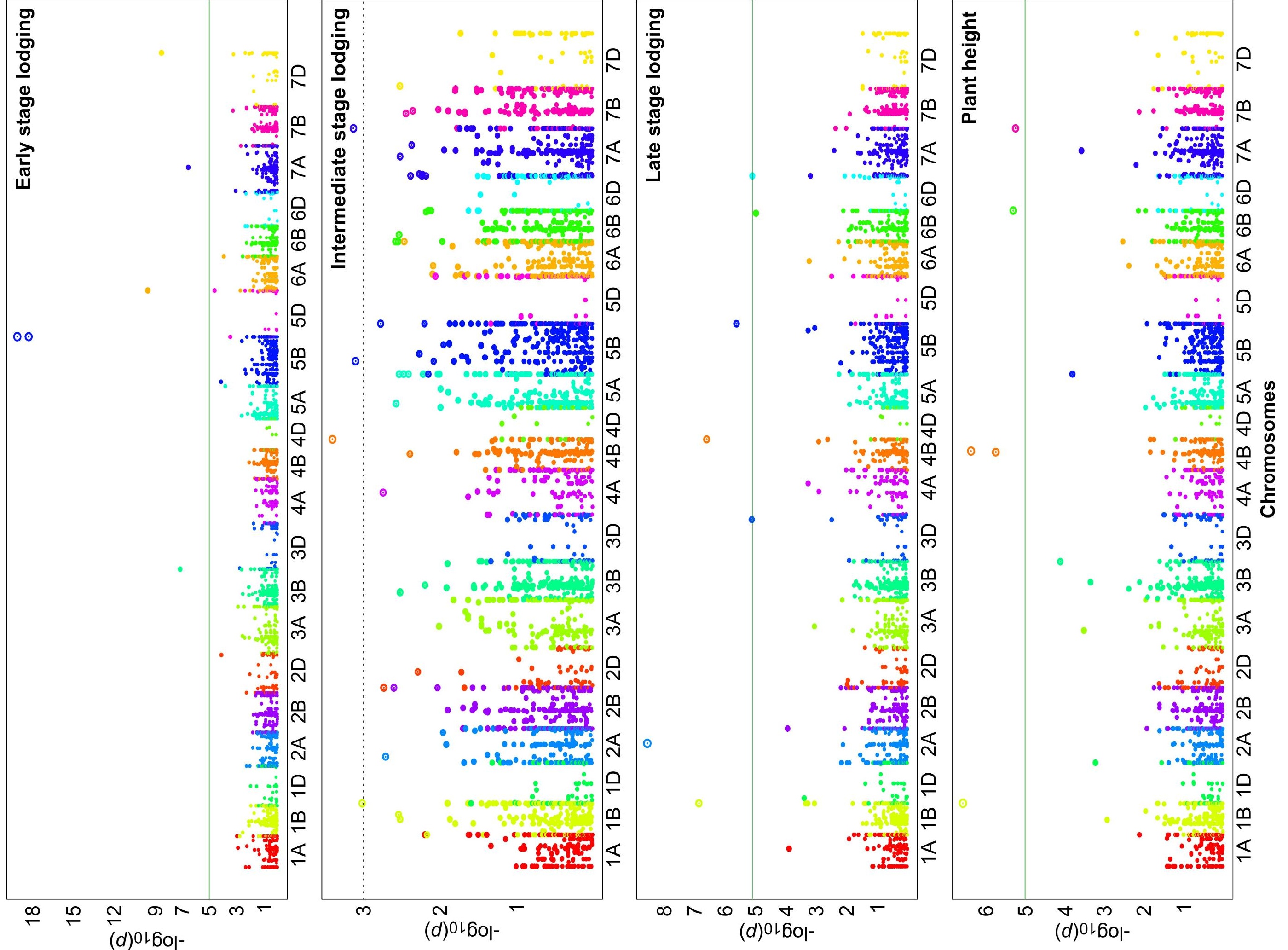




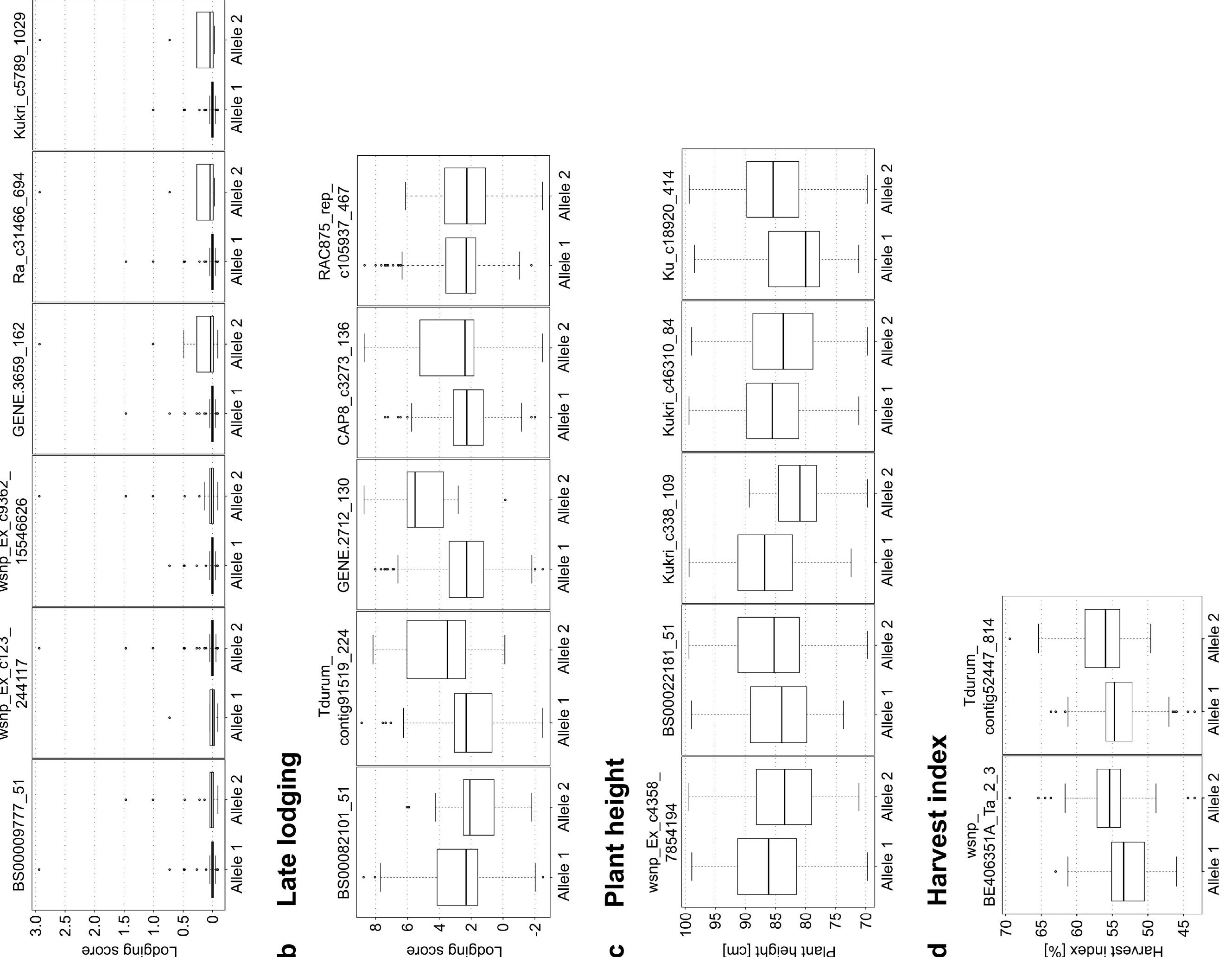




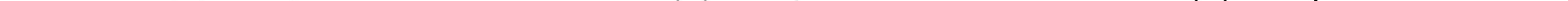




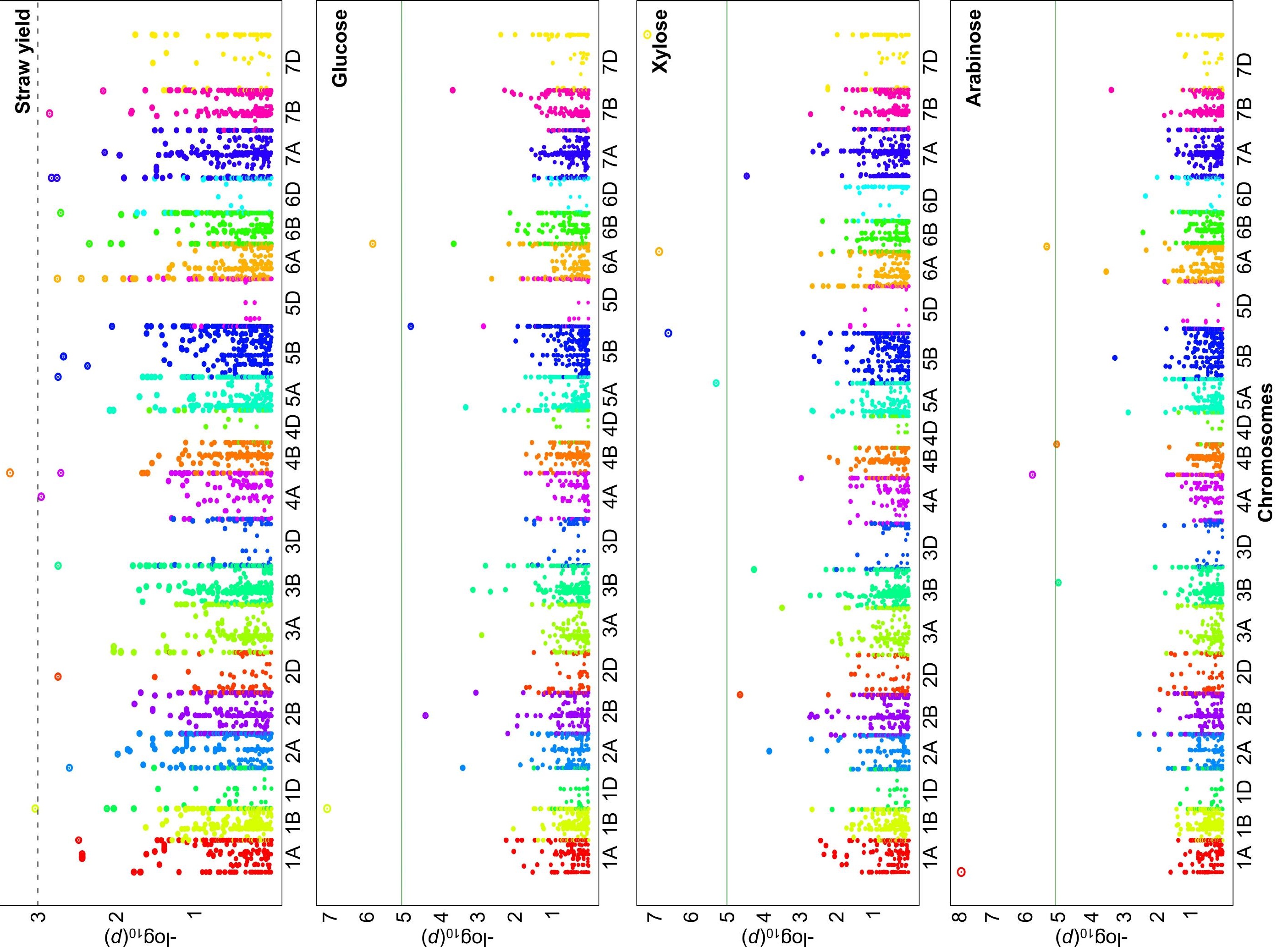


a STB

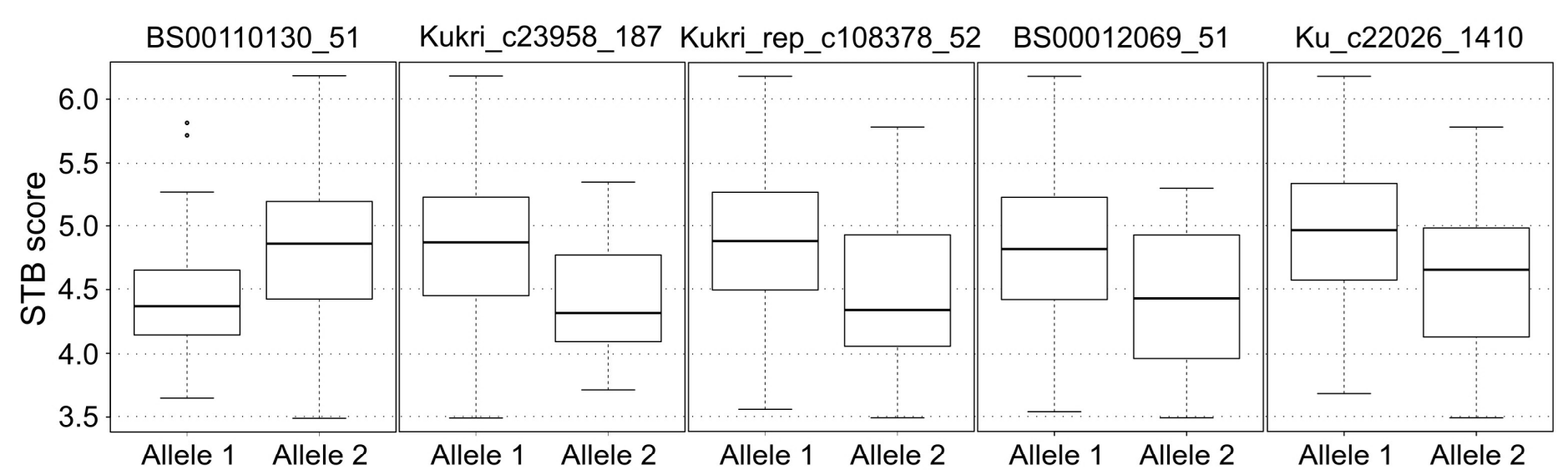

\section{b Glucose}

wsnp_Ex_c18384_ wsnp_Ex_c2236

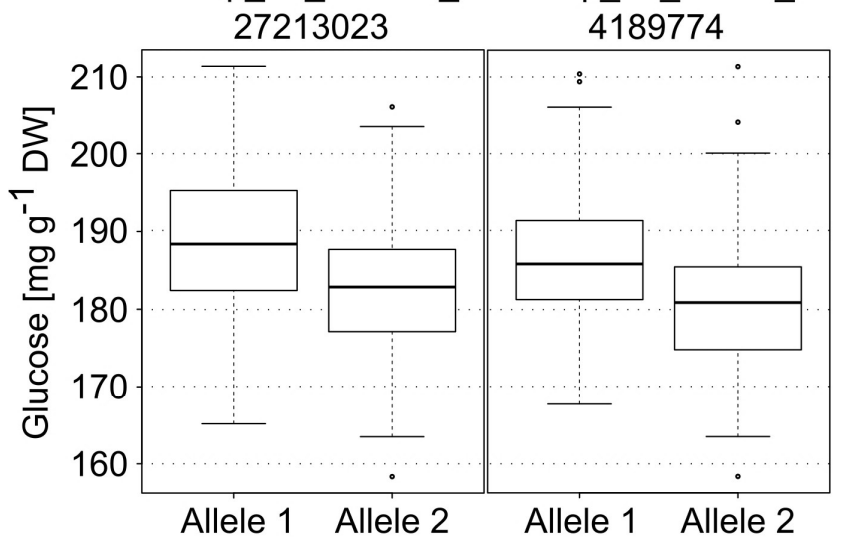

c Xylose

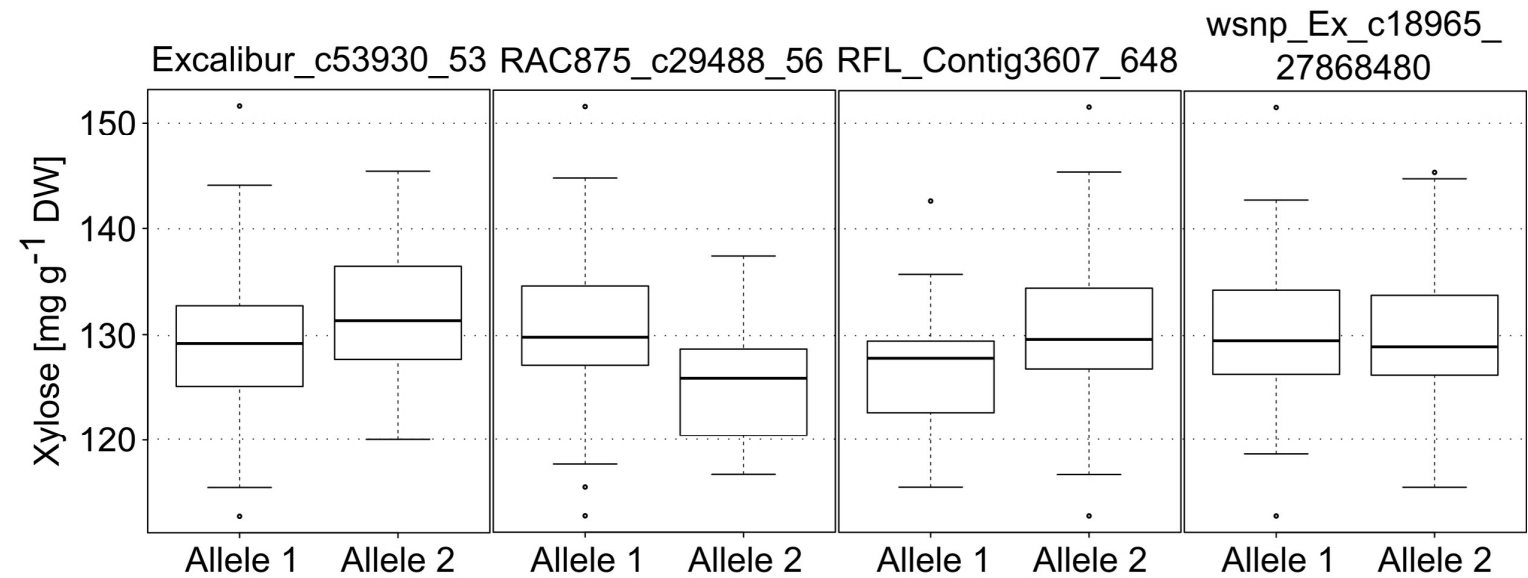

\section{d Arabinose}

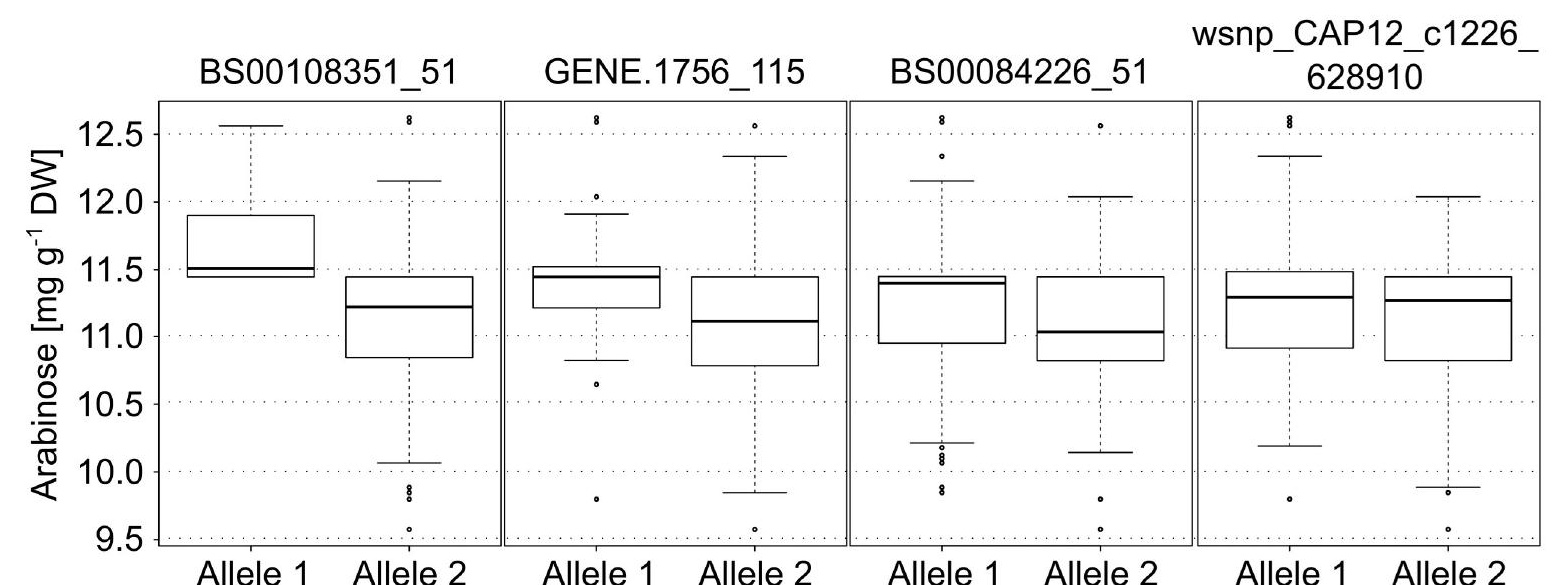




\section{Supplementary material}

\section{Breeding for dual-purpose wheat varieties using marker-trait associations for biomass yield and quality traits}

Theoretical and applied genetics

Pernille L. Malik ${ }^{\mathrm{a}}$, Luc Janss ${ }^{\mathrm{b}}$, Linda K. Nielsenc, Finn Borumc, Henning Jørgensen ${ }^{\mathrm{a}}$, Birger Eriksenc, Jan K. Schjoerring ${ }^{\mathrm{a}}$, Søren K. Rasmussen ${ }^{\mathrm{a}^{*}}$

aDepartment of Plant and Environmental Sciences, Faculty of Science, University of Copenhagen, Thorvaldsensvej 40, 1871 Frederiksberg, Denmark

bDepartment of Molecular Biology and Genetics, Aarhus University, Blichers Alle 20, 8830 Tjele, Denmark

'Sejet Plant Breeding, Nørremarksvej 67, 8700 Horsens, Denmark

*Corresponding author: skr@plen.ku.dk, Phone +45 35333436

Supplementary equations:

Equations for grain yield (eq. 1), straw yield (eq. 2 and 3) and harvest index (eq. 4) are given below.

$$
\begin{aligned}
& \operatorname{Grain}_{\text {yield }}\left[\frac{h k g}{h a}\right]=\frac{\operatorname{Grain}\left[\frac{\mathrm{kg}}{\text { parcel }}\right] *(100-15) * 100\left[\frac{\mathrm{hkg} * \mathrm{~m}^{2}}{\mathrm{~kg} * h a}\right]}{\frac{8.1\left[\frac{\mathrm{m}^{2}}{\text { parcel }}\right]}{100}} \\
& \text { Grain }_{\text {yield }}\left[\frac{\mathrm{t}}{\mathrm{ha}}\right]=\text { Grain }_{\text {yield }}\left[\frac{\mathrm{hkg}}{\mathrm{ha}}\right] * 0.1\left[\frac{\mathrm{t}}{\mathrm{hkg}}\right]
\end{aligned}
$$

The straw yield in $\mathrm{hkg} / \mathrm{ha}$ was obtained using the following equations:

$$
\begin{aligned}
& \operatorname{Straw}_{\text {waterloss }}[\%]=100-\left(\left(\frac{100}{\text { straw }_{\text {wet }}[g]}\right) * \operatorname{straw}_{\text {dry }}[g]\right)[\%] \\
& \operatorname{Straw}_{\text {yield }}[\mathrm{hKg} / \mathrm{ha}]=\frac{\text { straw }[\mathrm{kg}] *\left(100-\text { Straw }_{\text {waterloss }}[\%]\right) * 100}{\frac{8.1}{100}} \\
& \operatorname{Straw}_{\text {yield }}[t / h a]=\operatorname{Straw}_{\text {yield }}\left[\frac{\mathrm{hkg}}{h a}\right] * 0.1\left[\frac{t}{h k g}\right]
\end{aligned}
$$

The harvest index was calculated based on the mass of the grain yield and the mass of the biological yield (sum of grain yield and straw yield) by the following equation:

$$
\text { harvest index }[\%]=\frac{\text { economic yield }[\mathrm{hKg} / \mathrm{ha}]}{\text { biological yield }[\mathrm{hKg} / \mathrm{ha}]} * 100
$$


The conversion of sugar release from $\mathrm{mg} / \mathrm{g} \mathrm{DM}$ to t/ha was based on estimated means of sugar release and straw yield and was calculated as follows:

$$
\text { Sugar release }\left[\frac{t}{h a}\right]=\text { Straw }_{\text {yield }}\left[\frac{t D M}{h a}\right] * \text { sugar release }\left[\frac{m g}{g D M}\right] * 10^{-3}\left[\frac{g D M}{m g}\right]
$$

This equation was also used for converting the SNP effect for sugar release to $\mathrm{kg} / \mathrm{ha}$ and here was an average of the estimated means of straw yield used. 

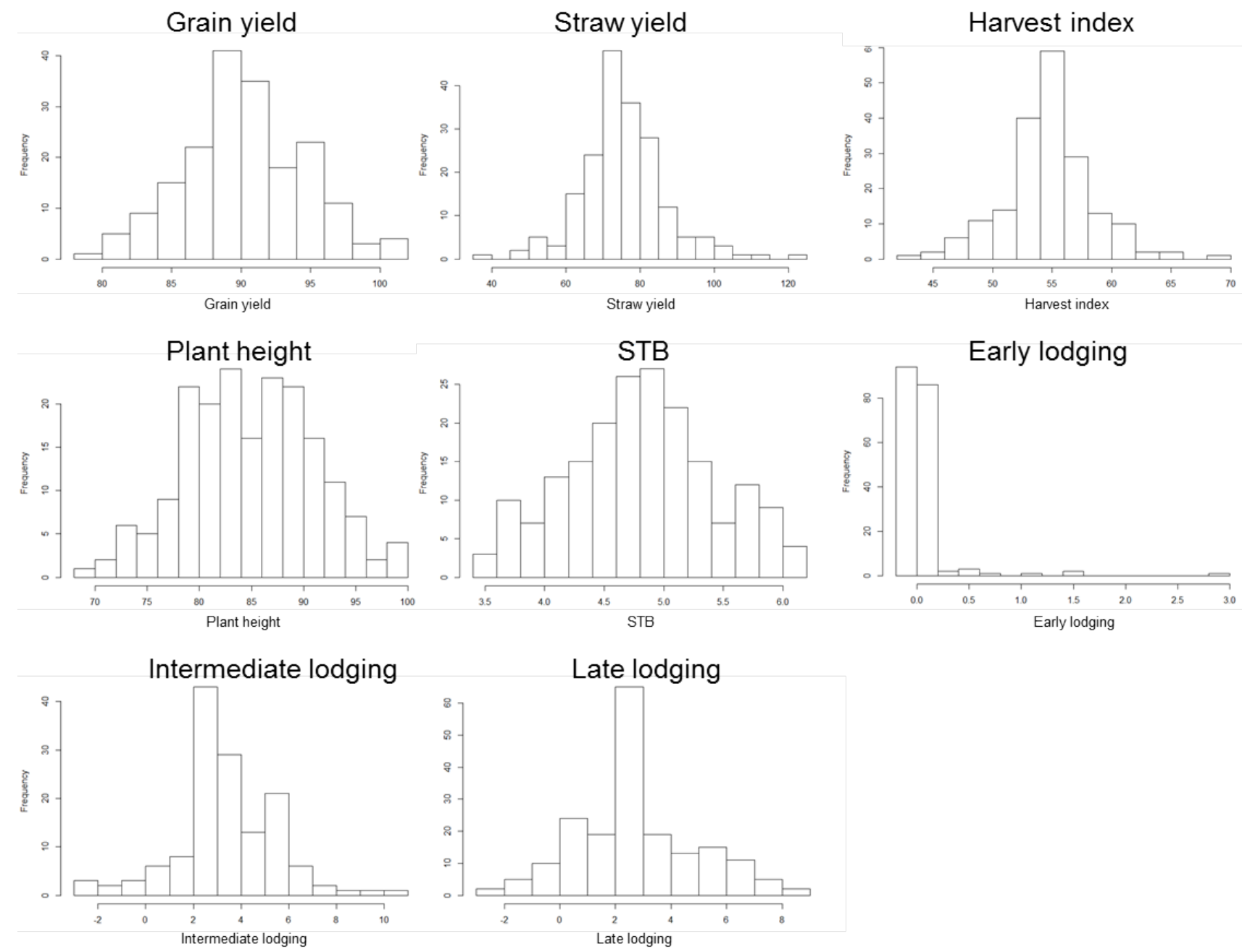

Figure S1: The distribution of phenotypic data from estimated means. As all the histograms show are all the phenotypic traits relatively normal distributed (with exception of the three stages of lodging) and there seem to be variation between cultivars. However only grain yield, plant height, STB and harvest index passed the Shapiro-Wilk normality test (p >0.001) (See Table S1). Septoria defines STB.

Table S1: Shapiro-Wilk normality test of estimated means

\begin{tabular}{|l|l|}
\hline Trait (estimated means) & p-value (Shapiro-Wilk normality test) \\
\hline Straw yield & $8.98 \times 19^{-5} *$ \\
\hline Plant height & 0.5013 \\
\hline Grain yield & 0.0724 \\
\hline
\end{tabular}




\begin{tabular}{|l|l|}
\hline Early lodging & $2.2 \times 10^{-16 *}$ \\
\hline Intermediate lodging & $6.575 \times 10^{-4} *$ \\
\hline Late lodging & $1.814 \times 10^{-4} *$ \\
\hline STB & 0.1172 \\
\hline Harvest index & $1.807 \times 10^{-3}$ \\
\hline Glucose & 0.6037 \\
\hline Xylose & 0.3269 \\
\hline Arabinose & $4.072 \times 10^{-5 *}$ \\
\hline
\end{tabular}

* defines p-values that are below 0.001 and show which traits that are not normal distributed. 

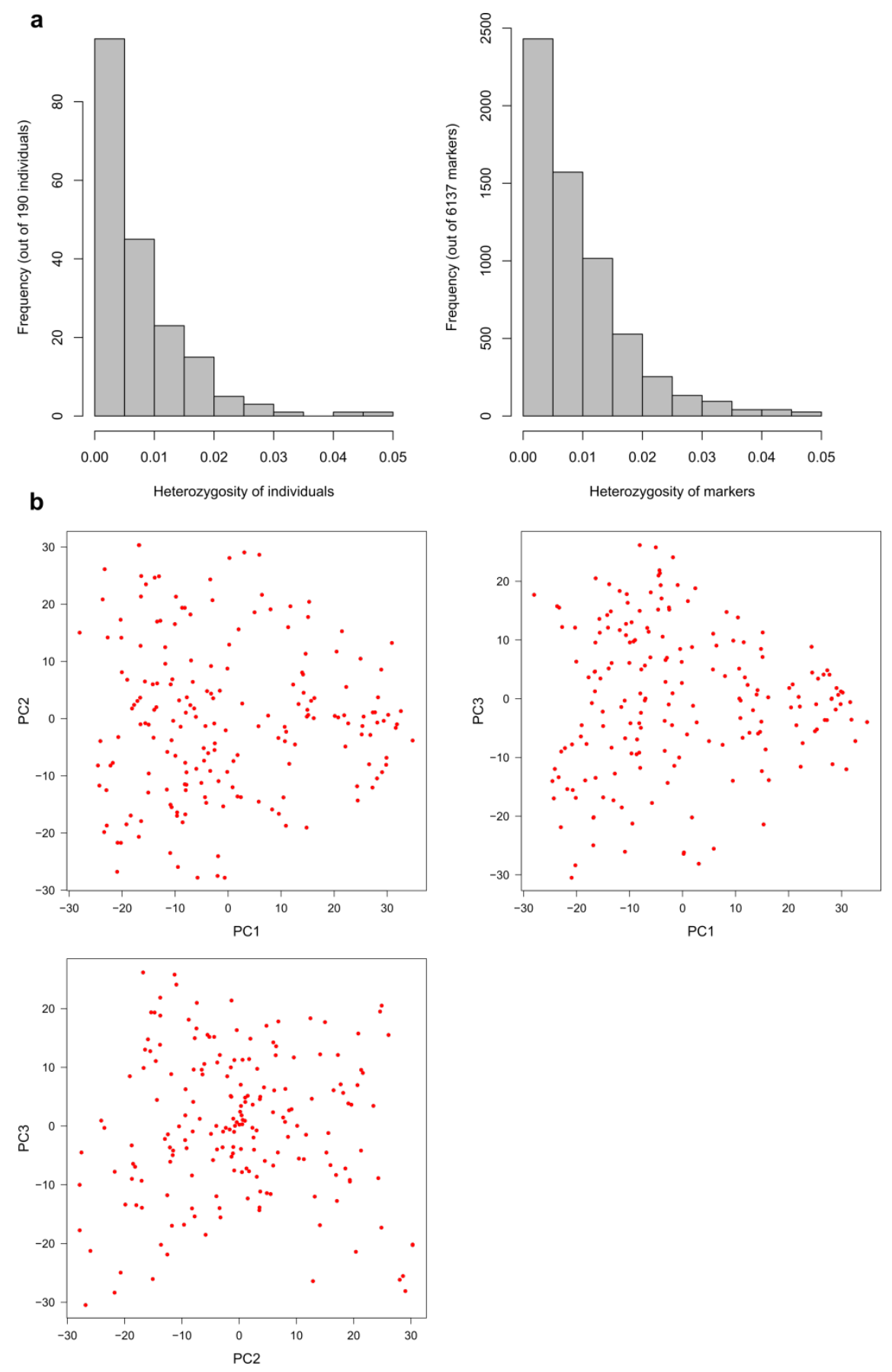

Figure S2: Population structure and heterozygosity. a) shows the heterozygosity of the 190 lines used in this study and the 6137 markers (including unknown markers) b) shows the distribution of the first 3 principal components plotted against each other. 
Straw yield

a

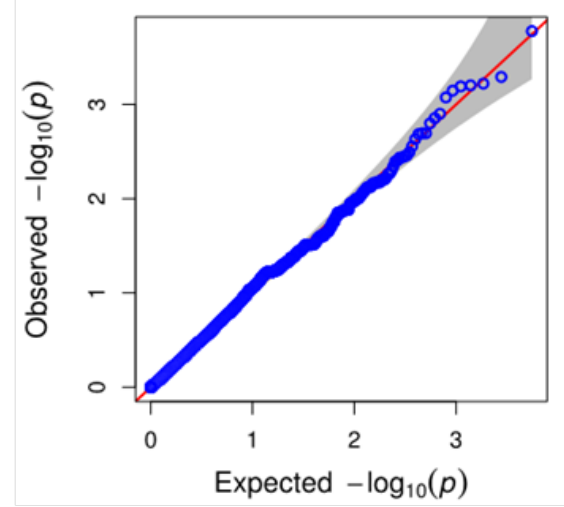

b

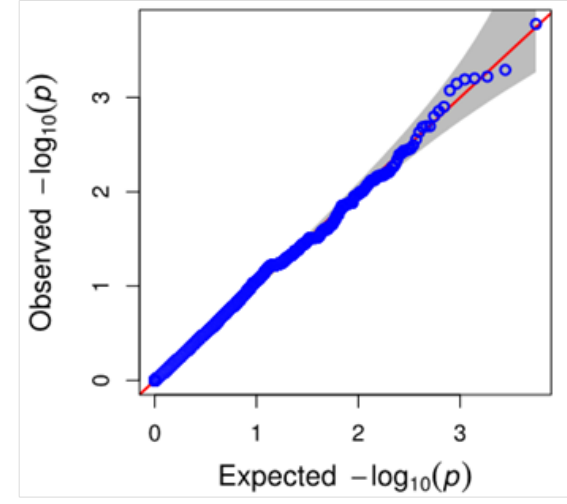

C

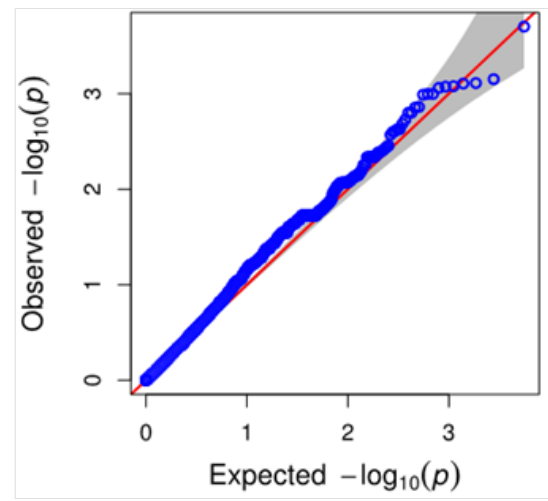

Grain yield
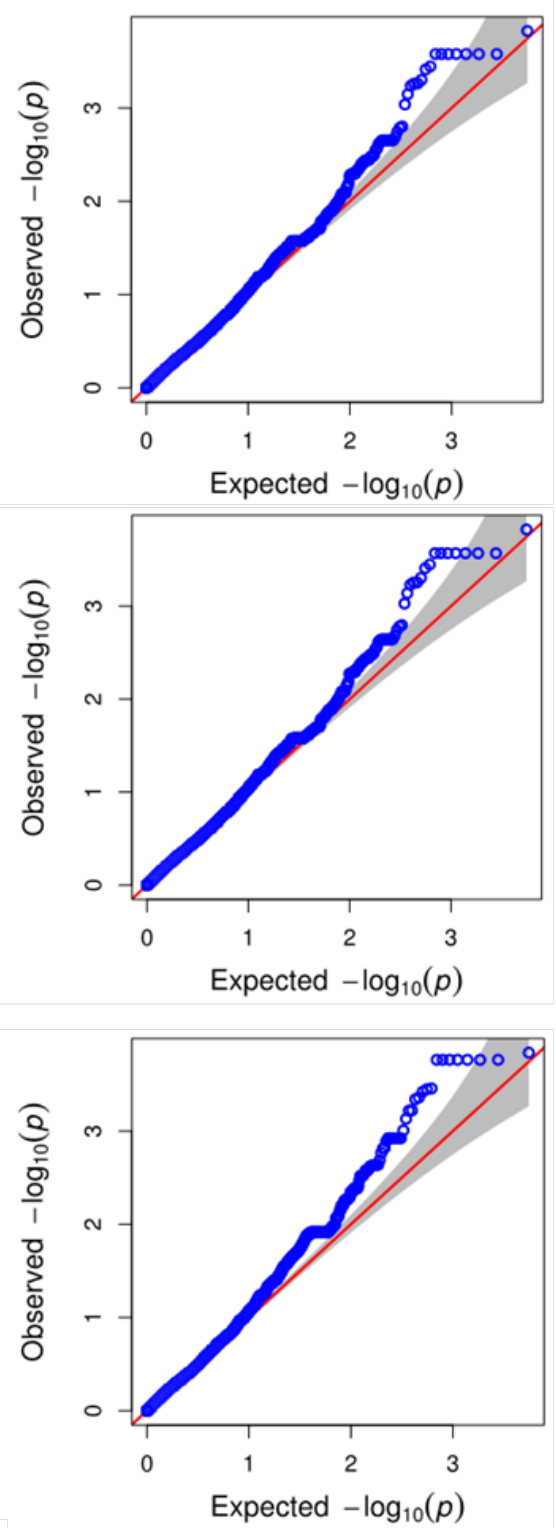

Plant height
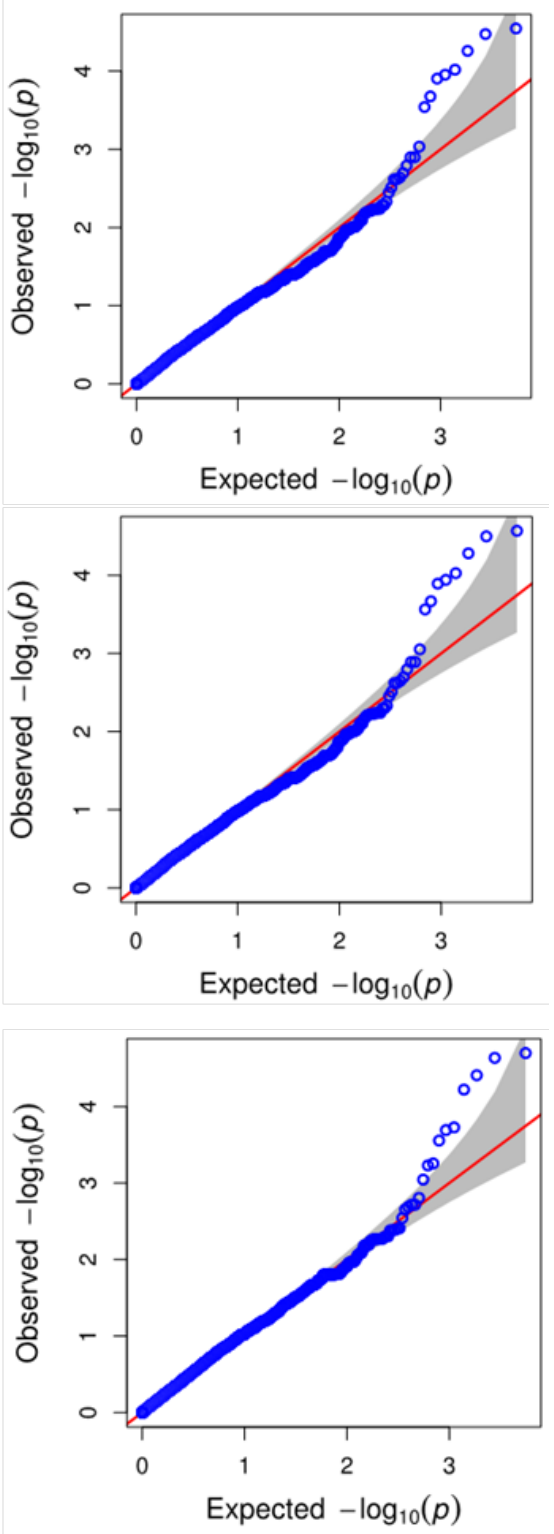

Figure S3: Comparison of QQ-plots between the three kinship algorithms available in standard GAPIT; a) Emma, b) VanRaden, and c) Loiselle. As an example was straw yield, grain yield and plant height chosen however, equivalent results were obtained for other traits. The QQ-plots are judged based on how well the plotted values follow the diagonal (red line) and drift off toward the end, as too much noise can indicate missing correction for population structure. 
Straw yield

a

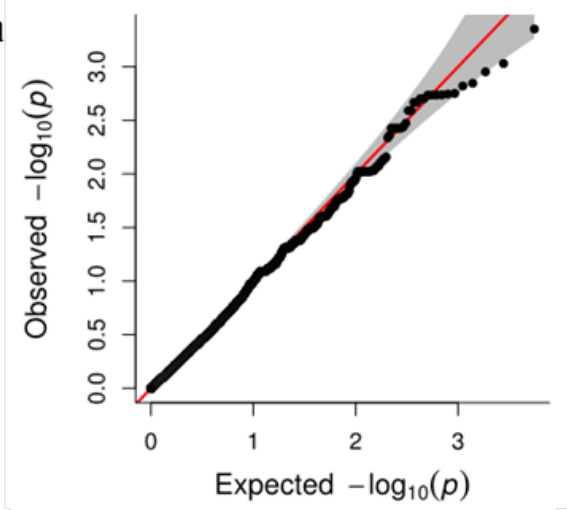

b

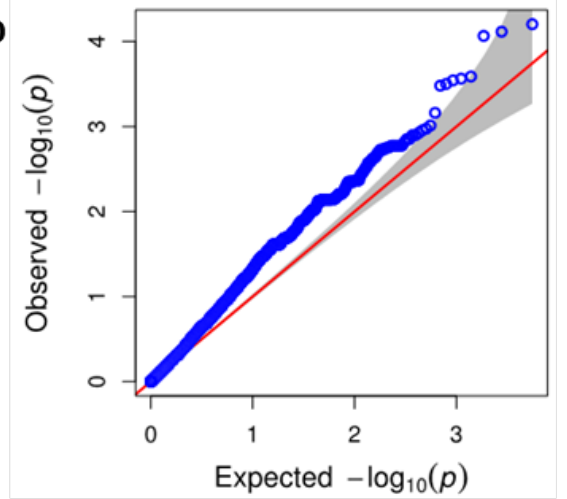

c

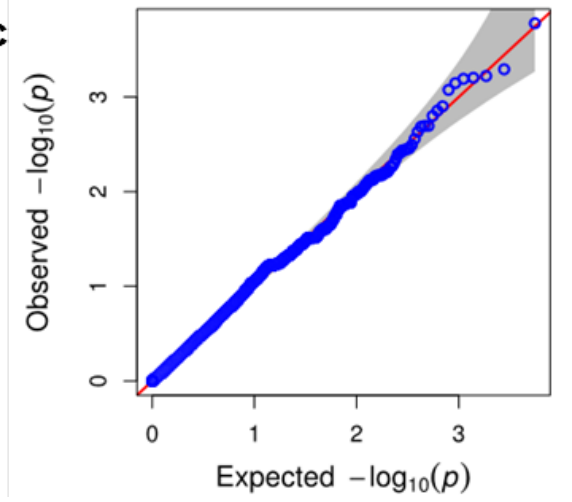

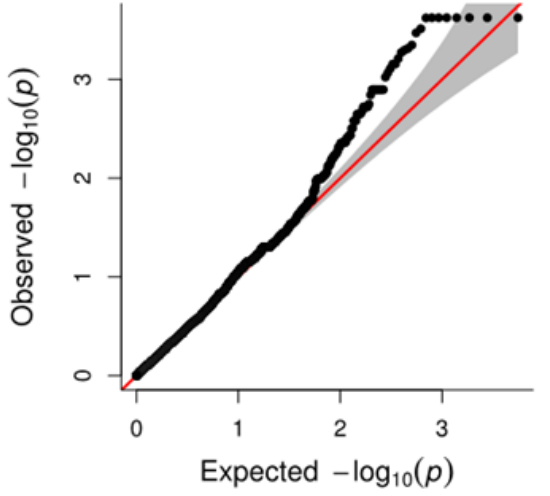
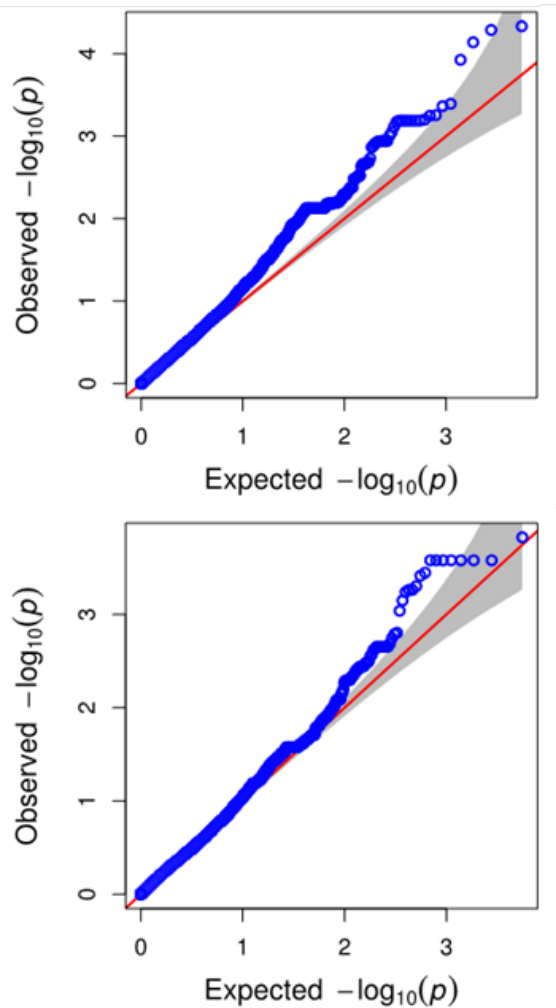

Plant height
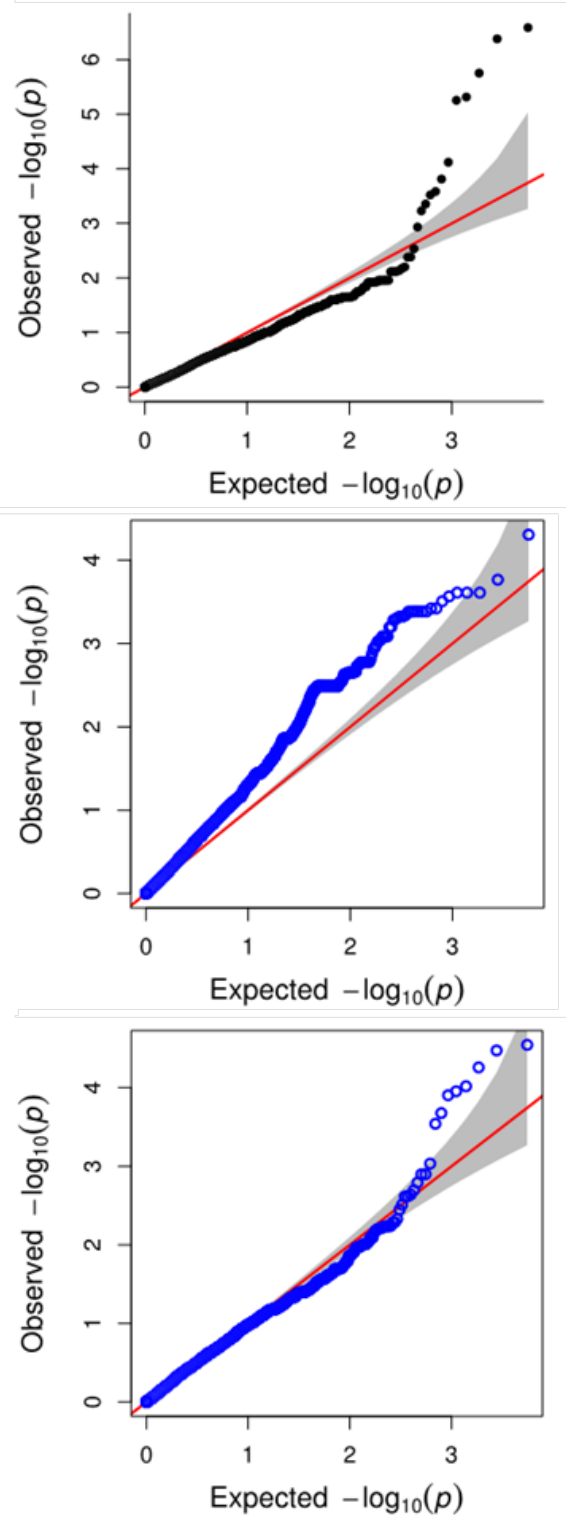

Figure S4: Comparing QQ-plots between a) FarmCPU b) SUPER and c) GAPIT-CMLM. Shown are QQ-plots for grain yield and straw yield as these traits behaved differently than the other traits and for plant height as serve as an example for all other traits. The QQ-plots are judged based on how well the plotted values follow the diagonal (red line) and drift off toward the end, as too much noise can indicate missing correction for population structure. 